\title{
Modular representations on some Riemann-Roch spaces of modular curves $X(N)$
}

\author{
David Joyner and Amy Ksir*†
}

$2-28-2005$

\begin{abstract}
We compute the $P S L(2, N)$-module structure of the RiemannRoch space $L(D)$, where $D$ is an invariant non-special divisor on the modular curve $X(N)$, with $N \geq 7$ prime. This depends on a computation of the ramification module, which we give explicitly. These results hold for characteristic $p$ if $X(N)$ has good reduction $\bmod p$ and $p$ does not divide the order of $\operatorname{PSL}(2, N)$. We give as examples the cases $N=7,11$, which were also computed using GAP]. Applications to AG codes associated to this curve are considered, and specific examples are computed using GAP and MAGMA.
\end{abstract}

\section{Contents}

1 Introduction 2

2 Modular curves 3

2.1 Ramification data . . . . . . . . . . . . . . . . . . 5

*Mathematics Dept, USNA, Annapolis, MD 21402,wdj@usna.edu and ksir@usna.edu

${ }^{\dagger}$ This is the expanded text of a talk given at the Algorithmic Algebraic Geometry session of the AMS Meeting in Altanta, GA, January 4-8, 2005. 
3 Representation theorv of $\operatorname{PSL}(2 . N) \quad 8$ 3.1 Brauer characters of some induced representations . . . . . . . 10

4 Induced characters 12

4.1 Induced characters from $H_{1} \ldots \ldots \ldots \ldots$. . . . . . . 12

4.2 Induced characters from $H_{2} \ldots \ldots \ldots \ldots \ldots$

4.3 Induced characters from $H_{3} \ldots \ldots \ldots \ldots$

5 Ramification module 19

6 Equivariant degree and Riemann-Roch space 25

$\begin{array}{lll}7 & \text { Examples } & 28\end{array}$

7.1 The case $N=7 \ldots \ldots \ldots \ldots$

7.2 The case $N=11 \ldots \ldots \ldots \ldots \ldots$

8 Application to codes 35

$9 \quad$ AG codes associated to $X(7) \quad 37$

10 Appendix: Tables for Theorem 8

11 Appendix: Ramification modules and GAP code 44

\section{Introduction}

The modular curve $X(N)$ has a natural action by the finite group $G=$ $P S L(2, N)$, with quotient $X(1)$. If $D$ is a $P S L(2, N)$-invariant divisor on $X(N)$, then there is a natural representation of $G$ on the Riemann-Roch space $L(D)$. In this paper, we give some results about the $P S L(2, N)$-module structure of the Riemann-Roch space $L(D)$, in the case where $N$ is prime and $N \geq 7$. If $D$ is non-special then a formula in Borne $[\mathrm{B}$ gives

$$
[L(D)]=\left(1-g_{X(1)}\right)[k[G]]+\left[\operatorname{deg}_{e q}(D)\right]-\left[\tilde{\Gamma}_{G}\right] .
$$

Here $g_{X(1)}$ is the genus of $X(1)$ (which is zero), square brackets denote the equivalence class of a representation of $G, \operatorname{deg}_{e q}(D)$ is the equivariant degree of $D$, and $\tilde{\Gamma}_{G}$ is the ramification module (these will be defined in sections 5 and (6). 
Our main result (see Theorem[1 in section [5) gives an explicit computation of the $G$-module structure of the ramification module $\tilde{\Gamma}_{G}$. We show it can be explicitly decomposed into irreducible $G$-modules where the multiplicity of an irreducible $G$-module $\pi$ in $\tilde{\Gamma}_{G}$ only depends on $\pi$ and the residue class of $N$ modulo 24. We then go on in section 6 to compute the equivariant degrees of $G$-invariant divisors on $X(N)$, and use these and Borne's formula to compute the $G$-module structure of the corresponding Riemann-Roch spaces.

If $K=\mathbb{Q}(G)$ denotes the abelian extension of $\mathbb{Q}$ generated by the character values of $G$ then $\mathcal{G}=\operatorname{Gal}(K / \mathbb{Q})$ acts on the set of irreducible representations of $G$. We call this the Galois action. In the case where $\tilde{\Gamma}_{G}$ is invariant under the Galois action, the authors have given a somewhat simpler formula for $\tilde{\Gamma}_{G}$ in JK]. In section 5 (see Theorem 9), we also prove that the ramification module is $\mathcal{G}$-invariant if and only if $N \equiv 1(\bmod 4)$.

As a corollary, it is an easy exercise now to compute explicitly the decomposition

$$
H^{1}(X(N), k)=H^{0}\left(X(N), \Omega^{1}\right) \oplus \overline{H^{0}\left(X(N), \Omega^{1}\right)}=L(K) \oplus \overline{L(K)}
$$

into irreducible $G$-modules, where $K$ is a canonical divisor. This was discussed in $\mathrm{KP}$ (over $k=\mathbb{C}$ ) and $\mathrm{Sc}$ (over the finite field $k=G F(N)$ ). Indeed, Schoen observes that the multiplicities of the irreducible representations occurring in $H^{1}(X(N), k)$ can be interpreted in terms of dimension of cusp forms and number of cusps on $X(N)$.

In section [7 we look at the examples $N=7,11$, using GAP to do many of the computations. In the last section, applications to AG codes associated to this curve are considered (MAGMA] was used to do some of these computations).

Notation: Throughout this paper, $N>5$ is a prime, $\mathbb{F}=G F(N)$ is the finite field with $N$ elements, and $G=\operatorname{PSL}(2, N)$.

Acknowledgements: We thank D. Prasad and R. Guralnick for enlightening correspondence, and in particular for the references [KP] and [Ja].

\section{Modular curves}

Let $H$ denote the complex upper half-plane, let $H^{*}=H \cup \mathbb{Q} \cup\{\infty\}$, and recall that $S L(2, \mathbb{Q})$ acts on $H^{*}$ by fractional linear transformations. Let 
$X(N)$ denote the modular curve defined over $\mathbb{Q}$ whose complex points are given by $\Gamma(N) \backslash H^{*}$, where

$$
\Gamma(N)=\left\{\left(\begin{array}{ll}
a & b \\
c & d
\end{array}\right) \in S L_{2}(\mathbb{Z}) \mid a-1 \equiv d-1 \equiv b \equiv c \equiv 0 \quad(\bmod N)\right\} .
$$

Throughout this paper, we will assume that $N$ is prime and $N>6$. In this case, the genus of $X(N)$ is given by the formula

$$
g=1+\frac{(N-6)\left(N^{2}-1\right)}{24} .
$$

For example, $X(7)$ is genus 3 and $X(11)$ is genus 26 .

Let $N$ be a prime and, for $j \in \mathbb{Z} / N \mathbb{Z}$, let $y_{j}$ be variables satisfying

$$
y_{j}+y_{-j}=0, \quad y_{a+b} y_{a-b} y_{c+d} y_{c-d}+y_{a+c} y_{a-c} y_{d+b} y_{d-b}+y_{a+d} y_{a-d} y_{b+c} y_{b-c}=0,
$$

for all $a, b, c, d \in \mathbb{Z} / N \mathbb{Z}$. These are Klein's equations for $X(N)$ (see Adler [A1] or Ritzenthaler [R1]).

Example 1 When $N=7$, this reduces to $y_{1}^{3} y_{2}-y_{2}^{3} y_{3}-y_{3}^{3} y_{1}=0$, the famous Klein quartic.

When $N=11$, the 20 equations which arise reduce to the 10 equations

$$
\begin{aligned}
-y_{1}^{2} y_{2} y_{3}+y_{2} y_{4} y_{5}^{2}+y_{3}^{2} y_{4} y_{5}=0 \\
-y_{1}^{3} y_{4}+y_{2} y_{4}^{3}-y_{3}^{3} y_{5}=0 \\
-y_{1} y_{3}^{3}-y_{1}^{3} y_{5}+y_{2}^{3} y_{4}=0 \\
-y_{1}^{2} y_{3} y_{4}+y_{1} y_{3} y_{5}^{2}+y_{2}^{2} y_{4} y_{5}=0 \\
-y_{1}^{2} y_{2} y_{5}+y_{1} y_{3} y_{4}^{2}-y_{2}^{2} y_{3} y_{5}=0 \\
y_{1}^{3} y_{2}-y_{3} y_{5}^{3}-y_{4}^{3} y_{5}=0 \\
y_{1} y_{5}^{3}-y_{2}^{3} y_{3}+y_{3}^{3} y_{4}=0 \\
-y_{1} y_{2}^{2} y_{4}+y_{1} y_{4}^{2} y_{5}+y_{2} y_{3}^{2} y_{5}=0 \\
y_{1} y_{2}^{3}+y_{2} y_{5}^{3}-y_{3} y_{4}^{3}=0 \\
y_{1} y_{2} y_{3}^{2}+y_{1} y_{4} y_{5}^{2}-y_{2} y_{3} y_{4}^{2}=0
\end{aligned}
$$

The curve $X(N)$ over a field $k$ parametrizes pairs of an elliptic curve over $k$ and a subgroup of order $N$ of the group structure on the curve. This can be extended to fields of positive characteristic, if $X(N)$ has good reduction. 
Since Klein's equations have integer coefficients they can also be extended to an arbitrary field $k$. However, Velu V] (see also Ritzenthaler [R3]) has shown that $X(N)$ has good reduction over fields of characteristic $p$ where $p$ does not divide $N$ (in our case, $p \neq N$, since $N$ is itself assumed to be a prime).

Let

$$
G=P S L_{2}(\mathbb{Z} / N \mathbb{Z}) \cong \overline{\Gamma(1)} / \overline{\Gamma(N)},
$$

where the overline denotes the image in $P S L_{2}(\mathbb{Z})$. This group acts on $X(N)$. (In characteristic 0 , see $[\mathbf{S}$, in characteristic $\ell>0$, see [R1].) When $N>2$ is prime, $|G|=N\left(N^{2}-1\right) / 2$. The quotient of $X(N)$ by the action of $P S L(2, N)$ is $X(1) \cong \mathbb{P}^{1}$.

Definition 2 When $X$ has good reduction to a finite field $k$ and, in addition, the characteristic $\ell$ of $k$ does not divide $|G|$, we say that $\ell$ is good.

If $k$ is a field of good characteristic, the automorphism group of $X(N)$ is known to be $P S L(2, N)$ BCG].

The action of $G=S L_{2}(\mathbb{Z} / N \mathbb{Z})$ on the set of points of the projective curve defined by Klein's equations is described in [R1] (see also [A2], [R2]). The element $g=\left(\begin{array}{ll}a & b \\ c & d\end{array}\right) \in G$ sends $\left(y_{j}\right)_{j \in \mathbb{Z} / N \mathbb{Z}} \in X(N)$ to $\left(\rho(g) y_{j}\right)_{j \in \mathbb{Z} / N \mathbb{Z}} \in$ $X(N)$, where

$$
\rho(g)\left(y_{j}\right)=\sum_{t \in \mathbb{Z} / N \mathbb{Z}} \zeta^{b\left(a j^{2}+2 j t c\right)+t^{2} c d} y_{a j+t c},
$$

where $\zeta$ denotes a primitive $N$-th root of unity in $k$.

Remark 1 When the formulas for the special cases $\rho\left(\begin{array}{cc}0 & 1 \\ -1 & 0\end{array}\right), \rho\left(\begin{array}{ll}1 & 1 \\ 0 & 1\end{array}\right)$, and $\rho\left(\begin{array}{cc}a & 0 \\ 0 & a^{-1}\end{array}\right)$, are written down separately, the similarity with the Weil representation for $S L_{2}(\mathbb{Z} / N \mathbb{Z}$ ) is striking (see also [A2]).

\subsection{Ramification data}

We will now consider the ramification data of the maps

$$
\phi_{N}: H^{*} \rightarrow X(N)
$$


and

$$
\psi_{N}: X(N) \rightarrow X(1) \cong \mathbb{P}^{1} .
$$

We will first study the characteristic zero case. We recall some facts from Shimura $\underline{\mathrm{S}}$.

We call a matrix $g=\left(\begin{array}{ll}a & b \\ c & d\end{array}\right) \in S L(2, \mathbb{Z})$ elliptic, parabolic, or $h y$ perbolic if $|\operatorname{tr}(g)|$ is $<2,=2$ and $g \neq I$, or $>2$, respectively. We call a point $z \in H^{*}$ elliptic, parabolic, or hyperbolic it it is fixed by such a matrix. Parabolic points are also called cuspidal points or cusps. Note that $g=\left(\begin{array}{ll}a & b \\ c & d\end{array}\right), c \neq 0$, fixes $z \in H$ if and only if

$$
z=\frac{a-d \pm \sqrt{(a+d)^{2}-4}}{2 c}
$$

(If $c=0$ then $g$ has no fixed points in $H$.)

If a group $G$ acts on a set $X$ then we denote by $G_{x}=\{g \in G \mid g(x)=x\}$ the stabilizer in $G$ of $x$.

\section{Lemma $3([S])$}

- If $z \in H^{*}$ is elliptic then $z$ belongs to the $S L_{2}(\mathbb{Z})$-orbit of $z_{1}=i$ or $z_{2}=\frac{1+\sqrt{3} i}{2}$.

- The stabilizer in $S L_{2}(\mathbb{Z})$ of $z_{1}$ is

$$
S L_{2}(\mathbb{Z})_{z_{1}}=\left\langle\left(\begin{array}{cc}
0 & 1 \\
-1 & 0
\end{array}\right)\right\rangle, \quad \Gamma(N)_{z_{1}}=\{I\},
$$

and of $z_{2}$ is

$$
S L_{2}(\mathbb{Z})_{z_{2}}=\left\langle\left(\begin{array}{cc}
0 & 1 \\
-1 & 1
\end{array}\right)\right\rangle, \quad \Gamma(N)_{z_{2}}=\{I\}
$$

- If $z \in H^{*}$ is parabolic then $z$ belongs to the $S L_{2}(\mathbb{Z})$-orbit of $\infty$. We have

$$
S L_{2}(\mathbb{Z})_{\infty}=\left\langle\left(\begin{array}{ll}
1 & 1 \\
0 & 1
\end{array}\right)\right\rangle, \quad \Gamma(N)_{\infty}=\left\langle\left(\begin{array}{cc}
1 & N \\
0 & 1
\end{array}\right)\right\rangle .
$$

- There are no hyperbolic points in $H^{*}$, 
The last part follows from (41). The others are proven in [S].

Lemma 4 ([S]) The ramification index of $\psi_{N}$ at $P=\phi_{N}(z)$ is $\left[\overline{\Gamma(1)}{ }_{z}\right.$ : $\left.\overline{\Gamma(N)_{z}}\right]$. The stabilizer at $P$ is

$$
G_{P}=\overline{\Gamma(1)}_{z} / \overline{\Gamma(N)}_{z} .
$$

In particular, we have the following.

- If $z=z_{1}$ then

$$
G_{P}=H_{1}=\left\langle\left(\begin{array}{cc}
0 & 1 \\
-1 & 0
\end{array}\right)\right\rangle
$$

is order 2 .

- If $z=z_{2}$ then

$$
G_{P}=H_{2}=\left\langle\left(\begin{array}{cc}
0 & 1 \\
-1 & 1
\end{array}\right)\right\rangle
$$

is order 3 .

- If $z=\infty$ then

$$
G_{P}=H_{3}=\left\langle\left(\begin{array}{ll}
1 & 1 \\
0 & 1
\end{array}\right)\right\rangle \cong \mathbb{Z} / N \mathbb{Z}
$$

is order $N$.

When $k=\mathbb{C}$, no other cyclic subgroups of $G$ occur as stabilizers.

Proposition 5 Assume $k$ is an algebraically closed field of good characteristic in the sense of Definition [2. If $H$ is a cyclic subgroup of $G=$ Aut $_{k}(X(N))=P S L_{2}(\mathbb{Z} / N \mathbb{Z})$ which is not of order 2 , 3, or $N$ then $H$ is not the stabilizer of any point on $X(N)$. Furthermore, there is only one orbit of points with stabilizer of order 2 , one orbit of points with stabilizer of order 3 , and one orbit of points with stabilizer of order $N$. 
proof: In the proof of Ritzenthaler [R1] Proposition 1.3 it is stated that $\phi_{N}$ is ramified over three points of indices 2, 3 and $N$. The Hurwitz(Riemann-Zeuthen) formula says that (see for example Hartshorne, Corollary IV.2.4 [H] $)$ :

$$
2 \cdot g(X(N))-2=-2 d+\operatorname{deg}(R)
$$

where $R$ is the ramification divisor

$$
R=\sum_{P \in X(N)} \text { length }\left(\Omega_{X(N) / X(1)}\right)_{P} \cdot P
$$

(Indeed, there are more explicit formulas for $\operatorname{deg}(R)$, since we are in the tamely ramified case.) Since the genus of $X(N)$ and of $X(1)$ do not depend on the characteristic, so we can conclude that the ramification divisor of $X(N) / \mathbb{C}$ has the same degree as the ramification divisor of $X(N) / k$. In other words, "deg $(R)$ over $\mathbb{C}$ " equals " $\operatorname{deg}(R)$ over $k$ ". Since all the summands in $\operatorname{deg}(R)$ are $\geq 0$, this is enough to show that no other cyclic subgroups of $G$ arise as stabilizers over $k$.

We call a cyclic subgroup of $G$ whose order is not $1,2,3$, or $N$ spurious. Spurious subgroups are not uncommon. Here is a general construction. If $N$ is prime, so $\mathbb{F}=\mathbb{Z} / N \mathbb{Z}$ is a field, let $\mathbb{E} / \mathbb{F}$ denote a quadratic field with norm $n m: \mathbb{E} \rightarrow \mathbb{F}$. The kernel of the norm map, $T=k e r(n m)$ embeds into $S L_{2}(\mathbb{F})$. Moreover, since $\mathbb{E}^{\times}$is cyclic, so is $T$, as well as all of its subgroups. It's known that $T$ has order $N+1$ since the norm map is surjective in this case.

Definition 6 At each point $P \in X(N)$, the ramification character $\theta_{P}$ is the character of the action of $G_{P}$ on the cotangent space to $X(N)$ at $P$.

\section{$3 \quad$ Representation theory of $\operatorname{PSL}(2, N)$}

We first consider the representation theory of $G$ over $\mathbb{C}$, following the treatment in $[\mathrm{FH}]$.

The group $P S L(2, N)$ has $3+(N-1) / 2$ conjugacy classes. Let $\varepsilon \in \mathbb{F}$ be a generator for the cyclic group $\mathbb{F}^{\times}$. Then each class will have a representative of one of the following forms: 


$$
\left(\begin{array}{ll}
1 & 0 \\
0 & 1
\end{array}\right),\left(\begin{array}{cc}
x & 0 \\
0 & x^{-1}
\end{array}\right),\left(\begin{array}{ll}
1 & 1 \\
0 & 1
\end{array}\right),\left(\begin{array}{ll}
1 & \varepsilon \\
0 & 1
\end{array}\right),\left(\begin{array}{cc}
x & \varepsilon y \\
y & x
\end{array}\right)
$$

The irreducible representations of $P S L(2, N)$ include the trivial representation 1 and one irreducible $V$ of dimension $N$. All but two of the others fall into two types: representations $W_{\alpha}$ of dimension $N+1$ ("principal series"), and $X_{\beta}$ of dimension $N-1$ ("discrete series"). The first type, $W_{\alpha}$, is labeled by a homomorphism $\alpha: \mathbb{F}^{\times} \rightarrow \mathbb{C}^{\times}$. The second type is indexed by a homomorphism $\beta: T \rightarrow \mathbb{C}^{\times}$, where $T$ is a cyclic subgroup of order $N+1$ of $\mathbb{F}(\sqrt{\varepsilon})^{\times}$. The characters of these are as follows:

\begin{tabular}{r||c|c|cc|c|c} 
& $\left(\begin{array}{ll}1 & 0 \\
0 & 1\end{array}\right)$ & $\left(\begin{array}{cc}x & 0 \\
0 & x^{-1}\end{array}\right)$ & $\left(\begin{array}{ll}1 & 1 \\
0 & 1\end{array}\right)$ & $\left(\begin{array}{ll}1 & \varepsilon \\
0 & 1\end{array}\right)$ & $\left(\begin{array}{cc}x & \varepsilon y \\
y & x\end{array}\right)$ \\
\hline \hline $\mathbf{1}$ & 1 & 1 & 1 & 1 & 1 \\
\hline$X_{\beta}$ & $N-1$ & 0 & -1 & -1 & $-\beta(x+\sqrt{\varepsilon} y)-\beta(x-\sqrt{\varepsilon} y)$ \\
\hline$V$ & $N$ & 1 & 0 & 0 & -1 \\
\hline$W_{\alpha}$ & $N+1$ & $\alpha(x)+\alpha\left(x^{-1}\right)$ & 1 & 1 & 0
\end{tabular}

Let $\tau$ denote a generator of $T$. Let $\zeta$ be a primitive $N$ th root of unity in C. Let $q$ and $q^{\prime}$ be defined by

$$
q=\sum_{\left(\frac{a}{N}\right)=1} \zeta^{a} \text { and } q^{\prime}=\sum_{\left(\frac{a}{N}\right)=-1} \zeta^{a}
$$

where the sums are over the quadratic residues and nonresidues $(\bmod N)$, respectively. If $N \equiv 1 \bmod 4$, then the "principal series" representation $W_{\alpha_{0}}$ corresponding to

$$
\begin{array}{ccc}
\alpha_{0}: \mathbb{F}^{\times} & \rightarrow & \mathbb{C}^{\times} \\
\varepsilon & \mapsto & -1
\end{array}
$$

is not irreducible, but splits into two irreducibles $W^{\prime}$ and $W^{\prime \prime}$, each of dimension $(N+1) / 2$. Their characters satisfy:

\begin{tabular}{c||c|c|cc|c} 
& $\left(\begin{array}{ll}1 & 0 \\
0 & 1\end{array}\right)$ & $\left(\begin{array}{cc}x & 0 \\
0 & x^{-1}\end{array}\right)$ & $\left(\begin{array}{ll}1 & 1 \\
0 & 1\end{array}\right)$ & $\left(\begin{array}{ll}1 & \varepsilon \\
0 & 1\end{array}\right)$ & $\left(\begin{array}{cc}x & \varepsilon y \\
y & x\end{array}\right)$ \\
\hline \hline$W^{\prime}$ & $\frac{N+1}{2}$ & $\alpha_{0}(x)$ & $1+q$ & $1+q^{\prime}$ & 0 \\
\hline$W^{\prime \prime}$ & $\frac{N+1}{2}$ & $\alpha_{0}(x)$ & $1+q^{\prime}$ & $1+q$ & 0
\end{tabular}


Similarly, if $N \equiv 3 \bmod 4$, then the "discrete series" representation $X_{\beta}$ corresponding to

$$
\begin{array}{ccc}
\beta_{0}: T & \rightarrow & \mathbb{C}^{\times} \\
\tau & \mapsto & -1
\end{array}
$$

sending the generator $\tau$ to -1 , splits into two irreducibles $X^{\prime}$ and $X^{\prime \prime}$, each of dimension $(N-1) / 2$. Their characters satisfy:

\begin{tabular}{c||c|c|cc|c} 
& $\left(\begin{array}{ll}1 & 0 \\
0 & 1\end{array}\right)$ & $\left(\begin{array}{cc}x & 0 \\
0 & x^{-1}\end{array}\right)$ & $\left(\begin{array}{ll}1 & 1 \\
0 & 1\end{array}\right)$ & $\left(\begin{array}{ll}1 & \varepsilon \\
0 & 1\end{array}\right)$ & $\left(\begin{array}{cc}x & \varepsilon y \\
y & x\end{array}\right)$ \\
\hline \hline$X^{\prime}$ & $\frac{N-1}{2}$ & 0 & $q$ & $q^{\prime}$ & $-\beta_{0}(x+y \sqrt{\varepsilon})$ \\
\hline$X^{\prime \prime}$ & $\frac{N-1}{2}$ & 0 & $q^{\prime}$ & $q$ & $-\beta_{0}(x+y \sqrt{\varepsilon})$
\end{tabular}

According to Janusz [Ja], the Schur index of each irreducible representation of $G$ is 1 .

The action of the Galois group $\mathcal{G}$ on the irreducible representations of $G$ is as follows. The character values lie in $\mathbb{Q}(\mu)$, where $\mu$ is a primitive $m^{\text {th }}$ root of unity and $m=N\left(N^{2}-1\right) / 4$. For each integer $j$ relatively prime to $m$, there is an element $\sigma_{j}$ of the Galois group $\mathcal{G}=\operatorname{Gal}(\mathbb{Q}(\mu) / \mathbb{Q})$ taking $\mu$ to $\mu^{j}$. This Galois group element will act on representations by taking a representation with character values $\left(a_{1}, \ldots, a_{n}\right)$ to a representation with character values $\left(\sigma_{j}\left(a_{1}\right), \ldots, \sigma_{j}\left(a_{n}\right)\right)$. Representations with rational character values will be fixed under this action.

Therefore the Galois group $\mathcal{G}$ will fix the trivial representation and the $N$-dimensional representation $V$. Its action preserves the set of $N-1$ dimensional "principal series" representations $X_{\beta}$, and the set of $N+1$ dimensional "discrete series" representations $W_{\alpha}$. In the case $N \equiv 1(\bmod 4)$, the Galois group will exchange the two $(N+1) / 2$-dimensional representations $W^{\prime}$ and $W^{\prime \prime}$; if $N \equiv 3(\bmod 4)$, the Galois group will exchange the two $(N-1) / 2$-dimensional representations $X^{\prime}$ and $X^{\prime \prime}$.

\subsection{Brauer characters of some induced representations}

Let $k$ be a field of characteristic $\ell$, assume $k$ contains a primitive $|G|$-th root of unity ${ }^{1}$, and assume that $\ell$ is good in the sense of (2). Because $\ell$ does not

\footnotetext{
${ }^{1}$ We really only need for $k$ to contain the character values of the irreducible $\bar{k}$ representations of $G$, where $\bar{k}$ denotes an algebraic closure of $k$.
} 
divide the order of $G$, the characters of $\mathbb{C}$-representations of $G$ are the same as the Brauer characters of $k$-representations of $G$. Some general remarks on the Brauer characters of induced modular representations follow.

Let $G$ be a finite group, let $H$ be a subgroup. Let $\sigma: H \rightarrow G L_{m}(k)$ be an $m$-dimensional representation. Let

$$
\sigma^{o}(g)= \begin{cases}\sigma(g), & g \in H, \\ 0, & g \in G-H .\end{cases}
$$

If $G=g_{1} H \cup g_{2} H \cup \ldots \cup g_{r} H$ is a disjoint union into cosets (where $g_{i} \in G$ and $g_{1}=1$ ) then let

$$
\pi=\operatorname{Ind}_{H}^{G} \sigma(g)=\left(\sigma^{o}\left(g_{i}^{-1} g g_{j}\right)\right)_{1 \leq i, j \leq r} .
$$

This $r m \times r m$ matrix is the induced representation of $\sigma$ to $G$. The trace of this representation is given by the $k$-valued class function

$$
\operatorname{tr} \pi(g)=\sum_{x \in G / H} \operatorname{tr} \sigma^{o}\left(x^{-1} g x\right)
$$

where we identify $G / H$ with $\left\{g_{1}, \ldots, g_{r}\right\}$.

Fix an embedding $k^{\times} \hookrightarrow \mathbb{C}^{\times}$(recall $k$ contains all the eigenvalues of each $\pi(g))$. Let $\chi_{\sigma}$ denote the Brauer character of $\sigma$ and let $\chi_{\pi}$ denote the Brauer character of $\pi$.

Proposition 7 If $H$ has no elements of order $\ell=\operatorname{char}(k)$ then

$$
\chi_{\pi}(g)=\sum_{x \in G / H} \chi_{\sigma}^{o}\left(x^{-1} g x\right),
$$

where $\chi_{\sigma}^{o}$ is the function $\chi_{\sigma}$ extended by 0 off of $H$.

This is well-known and the proof of this is straightforward, using only (6) and the definitions, so omitted.

In the next few sections, we will use induced characters to compute the $\operatorname{deg}_{e q}(D)$ and $\tilde{\Gamma}_{G}$. By the above proposition, such character computations will hold over $\mathbb{C}$ and in good positive characteristic. In particular, (14) and all the examples given in $\$ 7$ also hold in this case. 


\section{Induced characters}

To compute the $P S L(2, N)$-module structure of the Riemann-Roch space, we are interested in induced representations from the non-spurious cyclic subgroups

$$
H_{1}=\left\langle\left(\begin{array}{cc}
0 & 1 \\
-1 & 0
\end{array}\right)\right\rangle, \quad H_{2}=\left\langle\left(\begin{array}{cc}
0 & 1 \\
-1 & 1
\end{array}\right)\right\rangle, \quad H_{3}=\left\langle\left(\begin{array}{ll}
1 & 1 \\
0 & 1
\end{array}\right)\right\rangle
$$

of orders 2,3 , and $N$, respectively. We compute these by computing the restrictions of the irreducibles to these subgroups and using Frobenius reciprocity.

\subsection{Induced characters from $H_{1}$}

To compute the restrictions, we must find the conjugacy classes of $P S L(2, N)$ containing elements of these cyclic groups. For $H_{1}$ and $H_{2}$, these depend on $N$.

Define the number $i$ as follows. When $N \equiv 1(\bmod 4)$, let $i$ denote an element in $\mathbb{F}^{\times}$whose square is -1 (one can take $i=\varepsilon^{(N-1) / 4}$, where $\varepsilon$ is a generator of $\mathbb{F}^{\times}$). Then

$$
\left(\begin{array}{cc}
0 & 1 \\
-1 & 0
\end{array}\right) \text { is conjugate to }\left(\begin{array}{cc}
i & 0 \\
0 & i^{-1}
\end{array}\right) \text {. }
$$

When $N \equiv 3(\bmod 4)$, there is no square root of -1 in $\mathbb{F}$, so we pass to the quadratic extension and let $i=x+\sqrt{\varepsilon} y$ denote a square root of -1 in $\mathbb{F}(\sqrt{\varepsilon})^{\times}$. (If $\tau$ is a generator of the cyclic subgroup $T$ of order $N+1$ in $\mathbb{F}(\sqrt{\varepsilon})^{\times}$, we can take $i=\tau^{(N+1) / 4}$.) Then

$$
\left(\begin{array}{cc}
0 & 1 \\
-1 & 0
\end{array}\right) \text { is conjugate to }\left(\begin{array}{cc}
x & \varepsilon y \\
y & x
\end{array}\right) \text {. }
$$

Now let us compute the restrictions of the irreducible representations of $P S L(2, N)$ to $H_{1}$. We do this by examining the relevant columns of the character table of $P S L(2, N)$ from section 3 , and comparing them to the character table of the two element group $H_{1}$.

If $N \equiv 1(\bmod 4)$, the relevant columns read 


\begin{tabular}{r||c|c} 
& $\left(\begin{array}{ll}1 & 0 \\
0 & 1\end{array}\right)$ & $\left(\begin{array}{cc}i & 0 \\
0 & i^{-1}\end{array}\right)$ \\
\hline \hline $\mathbf{1}$ & 1 & 1 \\
\hline$W^{\prime}$ & $\frac{N+1}{2}$ & $\alpha_{0}(i)$ \\
\hline$W^{\prime \prime}$ & $\frac{N+1}{2}$ & $\alpha_{0}(i)$ \\
\hline$X_{\beta}$ & $N-1$ & 0 \\
\hline$V$ & $N$ & 1 \\
\hline$W_{\alpha}$ & $N+1$ & $\alpha(i)+\alpha\left(i^{-1}\right)$
\end{tabular}

Let $\theta_{1}$ be the nontrivial character of $H_{1}$. From these columns we see that the restricted representations are:

$$
\begin{aligned}
\operatorname{Res}_{H_{1}}^{G} \mathbf{1} & =\mathbf{1}_{H_{1}} \\
\operatorname{Res}_{H_{1}}^{G} W^{\prime} & =\frac{N-1}{4} \mathbf{1}_{H_{1}}+\frac{N-1}{4} \theta_{1}+\left\{\begin{array}{cll}
\mathbf{1}_{H_{1}} & \text { if } & \alpha_{0}(i)=1 \\
\theta_{1} & \text { if } & \alpha_{0}(i)=-1
\end{array}\right. \\
\operatorname{Res}_{H_{1}}^{G} W^{\prime \prime} & =\text { same } \\
\operatorname{Res}_{H_{1}}^{G} X_{\beta} & =\frac{N-1}{2} \mathbf{1}_{H_{1}}+\frac{N-1}{2} \theta_{1} \\
\operatorname{Res}_{H_{1}}^{G} V & =\frac{N+1}{2} \mathbf{1}_{H_{1}}+\frac{N-1}{2} \theta_{1} \\
\operatorname{Res}_{H_{1}}^{G} W_{\alpha} & =\frac{N-1}{2} \mathbf{1}_{H_{1}}+\frac{N-1}{2} \theta_{1}+\left\{\begin{array}{cll}
2 \mathbf{1}_{H_{1}} & \text { if } & \alpha(i)=1 \\
2 \theta_{1} & \text { if } & \alpha(i)=-1
\end{array}\right.
\end{aligned}
$$

The sign of $\alpha_{0}(i)$ depends on the equivalence class of $N(\bmod 8)$. Since $\alpha_{0}(\varepsilon)=-1$ and $i=\varepsilon^{(N-1) / 4}$, we will have $\alpha_{0}(i)=1$ if $(N-1) / 4$ is even $(N \equiv 1(\bmod 8))$ and $\alpha_{0}(i)=-1$ if $(N-1) / 4$ is odd $(N \equiv 5(\bmod 8))$. For the induced representation of the nontrivial character, we have, for $N \equiv 1$ $(\bmod 8)$,

$$
\begin{aligned}
\operatorname{Ind}_{H_{1}}^{G} \theta_{1}= & \frac{N-1}{4}\left(W^{\prime}+W^{\prime \prime}\right)+\frac{N-1}{2} \sum_{\beta} X_{\beta}+\frac{N-1}{2} V \\
& +\frac{N-1}{2} \sum_{\alpha(i)=1} W_{\alpha}+\frac{N+3}{2} \sum_{\alpha(i)=-1} W_{\alpha},
\end{aligned}
$$


and for $N \equiv 5(\bmod 8)$,

$$
\begin{aligned}
\operatorname{Ind}_{H_{1}}^{G} \theta_{1}= & \frac{N+3}{4}\left(W^{\prime}+W^{\prime \prime}\right)+\frac{N-1}{2} \sum_{\beta} X_{\beta}+\frac{N-1}{2} V \\
& +\frac{N-1}{2} \sum_{\alpha(i)=1} W_{\alpha}+\frac{N+3}{2} \sum_{\alpha(i)=-1} W_{\alpha} .
\end{aligned}
$$

Now let us compute the restricted representations for $N \equiv 3(\bmod 4)$. The relevant columns of the character table of $P S L(2, N)$ read

\begin{tabular}{r||c|c} 
& $\left(\begin{array}{ll}1 & 0 \\
0 & 1\end{array}\right)$ & $\left(\begin{array}{cc}x & \varepsilon y \\
y & x\end{array}\right)$ \\
\hline \hline $\mathbf{1}$ & 1 & 1 \\
\hline$X^{\prime}$ & $\frac{N-1}{2}$ & $-\beta_{0}(i)$ \\
\hline$X^{\prime \prime}$ & $\frac{N-1}{2}$ & $-\beta_{0}(i)$ \\
\hline$X_{\beta}$ & $N-1$ & $-\beta(i)-\beta\left(i^{-1}\right)$ \\
\hline$V$ & $N$ & -1 \\
\hline$W_{\alpha}$ & $N+1$ & 0
\end{tabular}

Therefore, the restricted representations are:

$$
\begin{aligned}
\operatorname{Res}_{H_{1}}^{G} \mathbf{1} & =\mathbf{1}_{H_{1}} \\
\operatorname{Res}_{H_{1}}^{G} X^{\prime} & =\frac{N-3}{4} \mathbf{1}_{H_{1}}+\frac{N-3}{4} \theta_{1}+\left\{\begin{array}{cl}
\mathbf{1}_{H_{1}} & \text { if } \beta_{0}(i)=-1 \\
\theta_{1} & \text { if } \beta_{0}(i)=1
\end{array}\right. \\
\operatorname{Res}_{H_{1}}^{G} X^{\prime \prime} & =\text { same } \\
\operatorname{Res}_{H_{1}}^{G} X_{\beta} & =\frac{N-3}{2} \mathbf{1}_{H_{1}}+\frac{N-3}{2} \theta_{1}+\left\{\begin{array}{cl}
2 \mathbf{1}_{H_{1}} & \text { if } \beta(i)=-1 \\
2 \theta_{1} & \text { if } \beta(i)=1
\end{array}\right. \\
\operatorname{Res}_{H_{1}}^{G} V & =\frac{N-1}{2} \mathbf{1}_{H_{1}}+\frac{N+1}{2} \theta_{1} \\
\operatorname{Res}_{H_{1}}^{G} W_{\alpha} & =\frac{N+1}{2} \mathbf{1}_{H_{1}}+\frac{N+1}{2} \theta_{1}
\end{aligned}
$$

As before, the sign of $\beta_{0}(i)$ in the representations induced from $X^{\prime}$ and $X^{\prime \prime}$ depends on the equivalence class of $N(\bmod 8)$. If $N \equiv 3(\bmod 8)$, then $\beta_{0}(i)=-1$ and if $N \equiv 7(\bmod 8)$, then $\beta_{0}(i)=1$. We get the following induced representations of the nontrivial character: if $N \equiv 3(\bmod 8)$, 


$$
\begin{aligned}
\operatorname{Ind}_{H_{1}}^{G} \theta_{1}= & \frac{N-3}{4}\left(X^{\prime}+X^{\prime \prime}\right)+\frac{N+1}{2} \sum_{\beta(i)=1} X_{\beta}+\frac{N-3}{2} \sum_{\beta(i)=-1} X_{\beta} \\
& +\frac{N+1}{2} V+\frac{N+1}{2} \sum_{\alpha} W_{\alpha}
\end{aligned}
$$

and if $N \equiv 7(\bmod 8)$,

$$
\begin{aligned}
\operatorname{Ind}_{H_{1}}^{G} \theta_{1}= & \frac{N+1}{4}\left(X^{\prime}+X^{\prime \prime}\right)+\frac{N+1}{2} \sum_{\beta(i)=1} X_{\beta}+\frac{N-3}{2} \sum_{\beta(i)=-1} X_{\beta} \\
& +\frac{N+1}{2} V+\frac{N+1}{2} \sum_{\alpha} W_{\alpha} .
\end{aligned}
$$

\subsection{Induced characters from $\mathrm{H}_{2}$}

Now we consider the induced characters from the cyclic group $H_{2}$ of order 3 . As in the case of $H_{1}$, the conjugacy classes of the elements of $H_{2}$ depend on $N$.

We define a number $\omega$ as follows. When $N \equiv 1(\bmod 3), \omega$ is a root of $x^{2}+1=x$ in $\mathbb{F}^{\times}$(one can take $\omega=\varepsilon^{(N-1) / 6}$ ). Note

$$
\left(\begin{array}{cc}
0 & 1 \\
-1 & 1
\end{array}\right) \text { is conjugate to }\left(\begin{array}{cc}
\omega & 0 \\
0 & \omega^{-1}
\end{array}\right)
$$

in $G$. When $N \equiv-1(\bmod 3), x+\sqrt{\varepsilon} y$ is a root of $x^{2}+1=x$ in $\mathbb{F}(\sqrt{\varepsilon})^{\times}$ (one can take $\omega=\tau^{(N+1) / 6}$ ). Note

$$
\left(\begin{array}{cc}
0 & 1 \\
-1 & 0
\end{array}\right) \text { is conjugate to }\left(\begin{array}{cc}
x & \varepsilon y \\
y & x
\end{array}\right)
$$

in $G$. In any case, the nontrivial elements of $H_{2}$ are $G$-conjugate.

Let $\theta_{2}$ and $\theta_{2}^{2}$ be the nontrivial characters of $H_{2}$. Again, to compute $\operatorname{Ind}_{\mathrm{H}_{2}}^{G} \theta_{2}$ and $\operatorname{Ind}_{\mathrm{H}_{2}}^{G} \theta_{2}^{2}$, we compute the restrictions of the irreducible characters of $G$ and use Frobenius reciprocity.

If $N \equiv 1(\bmod 3)$, the relevant columns of the character table of $G=$ $P S L(2, N)$ read 


\begin{tabular}{r||c|c} 
& $\left(\begin{array}{ll}1 & 0 \\
0 & 1\end{array}\right)$ & $\left(\begin{array}{cc}\omega & 0 \\
0 & \omega^{-1}\end{array}\right)$ \\
\hline \hline $\mathbf{1}$ & 1 & 1 \\
\hline$X^{\prime}$ & $\frac{N-1}{2}$ & 0 \\
\hline$X^{\prime \prime}$ & $\frac{N-1}{2}$ & 0 \\
\hline$W^{\prime}$ & $\frac{N+1}{2}$ & $\alpha_{0}(\omega)$ \\
\hline$W^{\prime \prime}$ & $\frac{N+1}{2}$ & $\alpha_{0}(\omega)$ \\
\hline$X_{\beta}$ & $N-1$ & 0 \\
\hline$V$ & $N$ & 1 \\
\hline$W_{\alpha}$ & $N+1$ & $\alpha(\omega)+\alpha\left(\omega^{-1}\right)$
\end{tabular}

where the character table contains $W^{\prime}$ and $W^{\prime \prime}$ if $N \equiv 1(\bmod 4)$, and $X^{\prime}$ and $X^{\prime \prime}$ if $N \equiv 3(\bmod 4)$. If $N \equiv 1(\bmod 4)$, the value of $\alpha_{0}(\omega)$ is $(-1)^{(N-1) / 6}$ and $\frac{N-1}{6}$ is even, so $\alpha_{0}(\omega)=1$. Therefore, the restricted representations are:

$$
\begin{aligned}
& \operatorname{Res}_{H_{2}}^{G} \mathbf{1}=\mathbf{1}_{H_{2}} \\
& \operatorname{Res}_{H_{2}}^{G} X^{\prime}=\frac{N-1}{6}\left(\mathbf{1}_{H_{2}}+\theta_{2}+\theta_{2}^{2}\right) \\
& \operatorname{Res}_{H_{2}}^{G} X^{\prime \prime}=\text { same } \\
& \operatorname{Res}_{H_{2}}^{G} W^{\prime}=\frac{N-1}{6}\left(\mathbf{1}_{H_{2}}+\theta_{2}+\theta_{2}^{2}\right)+\mathbf{1}_{H_{2}} \\
& \operatorname{Res}_{H_{2}}^{G} W^{\prime \prime}=\text { same } \\
& \operatorname{Res}_{H_{2}}^{G} X_{\beta}=\frac{N-1}{3}\left(\mathbf{1}_{H_{2}}+\theta_{2}+\theta_{2}^{2}\right) \\
& \operatorname{Res}_{H_{2}}^{G} V=\frac{N-1}{3}\left(\mathbf{1}_{H_{2}}+\theta_{2}+\theta_{2}^{2}\right)+\mathbf{1}_{H_{2}} \\
& \operatorname{Res}_{H_{2}}^{G} W_{\alpha}=\frac{N-1}{3}\left(\mathbf{1}_{H_{2}}+\theta_{2}+\theta_{2}^{2}\right)+\left\{\begin{array}{ccc}
2 \mathbf{1}_{H_{2}} & \text { if } & \alpha(\omega)=1 \\
\theta_{2}+\theta_{2}^{2} & \text { if } & \alpha(\omega) \neq 1
\end{array}\right. \\
& \operatorname{Ind}_{H_{2}}^{G} \theta_{2}=\operatorname{Ind}_{H_{2}}^{G} \theta_{2}^{2}=\frac{N-1}{3} \sum_{\beta} X_{\beta}+\frac{N-1}{3} V \\
& +\frac{N-1}{3} \sum_{\alpha(\omega)=1} W_{\alpha}+\frac{N+2}{3} \sum_{\alpha(\omega) \neq 1} W_{\alpha} \\
& +\frac{N-1}{6}\left\{\begin{array}{ccc}
W^{\prime}+W^{\prime \prime} & \text { if } & N \equiv 1 \quad(\bmod 12) \\
X^{\prime}+X^{\prime \prime} & \text { if } & N \equiv 7 \quad(\bmod 12)
\end{array}\right.
\end{aligned}
$$


Assume next $N \equiv 2(\bmod 3)$. Again, the character table contains $W^{\prime}$ and $W^{\prime \prime}$ if $N \equiv 1(\bmod 4)($ so $N \equiv 5(\bmod 12))$, and $X^{\prime}$ and $X^{\prime \prime}$ if $N \equiv 3$ $(\bmod 4)($ so $N \equiv 11(\bmod 12))$. The relevant columns of the character table of $G$ are as follows. Note that for the irreducibles $X^{\prime}$ and $X^{\prime \prime}$ (in the case $N \equiv 11(\bmod 12))$, the character value depends on $\beta_{0}(\omega)$, where $\beta_{0}$ is the character of the cyclic group of order $N+1$ sending a generator to -1 . In this case, $\omega=\tau^{\frac{N+1}{6}}$, and $\frac{N+1}{6}$ is even, so $\beta_{0}(\omega)=1$.

\begin{tabular}{r||c|c} 
& $\left(\begin{array}{ll}1 & 0 \\
0 & 1\end{array}\right)$ & $\left(\begin{array}{cc}x & \varepsilon y \\
y & x\end{array}\right)$ \\
\hline \hline 1 & 1 & 1 \\
\hline$X^{\prime}$ & $\frac{N-1}{2}$ & -1 \\
\hline$X^{\prime \prime}$ & $\frac{N-1}{2}$ & -1 \\
\hline$W^{\prime}$ & $\frac{N+1}{2}$ & 0 \\
\hline$W^{\prime \prime}$ & $\frac{N+1}{2}$ & 0 \\
\hline$X_{\beta}$ & $N-1$ & $-\beta(\omega)-\beta\left(\omega^{-1}\right)$ \\
\hline$V$ & $N$ & -1 \\
\hline$W_{\alpha}$ & $N+1$ & 0
\end{tabular}

Therefore, we see that when $N \equiv 2(\bmod 3)$, the restricted representations are:

$$
\begin{aligned}
\operatorname{Res}_{H_{2}}^{G} \mathbf{1} & =\mathbf{1}_{H_{2}} \\
\operatorname{Res}_{H_{2}}^{G} X^{\prime} & =\frac{N-5}{6}\left(\mathbf{1}_{H_{2}}+\theta_{2}+\theta_{2}^{2}\right)+\theta_{2}+\theta_{2}^{2} \\
\operatorname{Res}_{H_{2}}^{G} X^{\prime \prime} & =\text { same } \\
\operatorname{Res}_{H_{2}}^{G} W^{\prime} & =\frac{N+1}{6}\left(\mathbf{1}_{H_{2}}+\theta_{2}+\theta_{2}^{2}\right) \\
\operatorname{Res}_{H_{2}}^{G} W^{\prime \prime} & =\text { same } \\
\operatorname{Res}_{H_{2}}^{G} X_{\beta} & =\frac{N-5}{3}\left(\mathbf{1}_{H_{2}}+\theta_{2}+\theta_{2}^{2}\right)+\left\{\begin{array}{cc}
2\left(\theta_{2}+\theta_{2}^{2}\right) & \text { if } \beta(\omega)=1 \\
2 \mathbf{1}_{H_{2}}+\theta_{2}+\theta_{2}^{2} & \text { if } \beta(\omega) \neq 1
\end{array}\right. \\
\operatorname{Res}_{H_{2}}^{G} V & =\frac{N-2}{3}\left(\mathbf{1}_{H_{2}}+\theta_{2}+\theta_{2}^{2}\right)+\theta_{2}+\theta_{2}^{2} \\
\operatorname{Res}_{H_{2}}^{G} W_{\alpha} & =\frac{N+1}{3}\left(\mathbf{1}_{H_{2}}+\theta_{2}+\theta_{2}^{2}\right)
\end{aligned}
$$


And the induced representations for $N \equiv 2(\bmod 3)$ are:

$$
\begin{aligned}
& \operatorname{Ind}_{H_{2}}^{G} \theta_{2}=\operatorname{Ind}_{H_{2}}^{G} \theta_{2}^{2}=\frac{N+1}{3} \sum_{\beta} X_{\beta}+\frac{N-2}{3} \sum_{\beta} X_{\beta}+\frac{N+1}{3} \sum_{\alpha} W_{\alpha} \\
& +\frac{N+1}{3} V+\frac{N+1}{6}\left\{\begin{array}{ccl}
W^{\prime}+W^{\prime \prime} & \text { if } & N \equiv 5 \quad(\bmod 12) \\
X^{\prime}+X^{\prime \prime} & \text { if } & N \equiv 11 \quad(\bmod 12)
\end{array}\right.
\end{aligned}
$$

\subsection{Induced characters from $H_{3}$}

Now we consider the induced characters from $H_{3}$, the cyclic group of order $N$. For the restrictions of the irreducible characters of $\operatorname{PSL}(2, N)$ to $H_{3}$, the relevant columns of the character table of $G=P S L(2, N)$ are as follows. As before, the representations $W^{\prime}$ and $W^{\prime \prime}$ appear if and only if $N \equiv 1(\bmod 4)$, and $X^{\prime}$ and $X^{\prime \prime}$ appear if and only if $N \equiv 3(\bmod 4)$.

\begin{tabular}{r||c|cc} 
& $\left(\begin{array}{ll}1 & 0 \\
0 & 1\end{array}\right)$ & $\left(\begin{array}{ll}1 & 1 \\
0 & 1\end{array}\right)$ & $\left(\begin{array}{ll}1 & \varepsilon \\
0 & 1\end{array}\right)$ \\
\hline \hline $\mathbf{1}$ & 1 & 1 & 1 \\
\hline$X^{\prime}$ & $\frac{N-1}{2}$ & $q$ & $q^{\prime}$ \\
\hline$X^{\prime \prime}$ & $\frac{N-1}{2}$ & $q^{\prime}$ & $q$ \\
\hline$W^{\prime}$ & $\frac{N+1}{2}$ & $1+q$ & $1+q^{\prime}$ \\
\hline$W^{\prime \prime}$ & $\frac{N+1}{2}$ & $1+q^{\prime}$ & $1+q$ \\
\hline$X_{\beta}$ & $N-1$ & -1 & -1 \\
\hline$V$ & $N$ & 0 & 0 \\
\hline$W_{\alpha}$ & $N+1$ & 1 & 1
\end{tabular}

Recall

$$
q=\sum_{\left(\frac{a}{N}\right)=1} \zeta^{a} \text { and } q^{\prime}=\sum_{\left(\frac{a}{N}\right)=-1} \zeta^{a} .
$$

Now let $\theta_{3}$ be the nontrivial character of $H_{3}$ such that

$$
\theta_{3}\left(\begin{array}{ll}
1 & 1 \\
0 & 1
\end{array}\right)=\zeta .
$$


The restricted representations are:

$$
\begin{aligned}
\operatorname{Res}_{H_{3}}^{G} \mathbf{1} & =\mathbf{1}_{H_{3}} \\
\operatorname{Res}_{H_{3}}^{G} X^{\prime} & =\sum_{\left(\frac{k}{n}\right)=1} \theta_{3}^{k} \\
\operatorname{Res}_{H_{3}}^{G} X^{\prime \prime} & =\sum_{\left(\frac{k}{n}\right)=-1} \theta_{3}^{k} \\
\operatorname{Res}_{H_{3}}^{G} W^{\prime} & =\mathbf{1}_{H_{3}}+\sum_{\left(\frac{k}{n}\right)=1} \theta_{3}^{k} \\
\operatorname{Res}_{H_{3}}^{G} W^{\prime \prime} & =\mathbf{1}_{H_{3}}+\sum_{\left(\frac{k}{n}\right)=-1} \theta_{3}^{k} \\
\operatorname{Res}_{H_{3}}^{G} X_{\beta} & =\sum_{k=1}^{N-1} \theta_{3}^{k} \\
\operatorname{Res}_{H_{3}}^{G} V & =\mathbf{1}_{H_{3}}+\sum_{k=1}^{N-1} \theta_{3}^{k} \\
\operatorname{Res}_{H_{3}}^{G} W_{\alpha} & =2 \mathbf{1}_{H_{3}}+\sum_{k=1}^{N-1} \theta_{3}^{k}
\end{aligned}
$$

Threrefore,

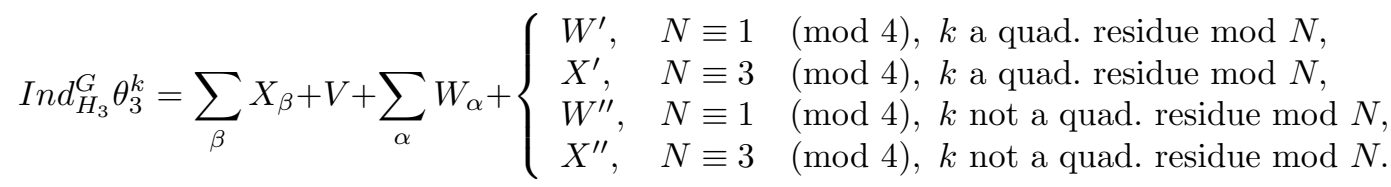

\section{Ramification module}

For the remainder of this paper, let $G^{*}$ denote the set of equivalence classes of irreducible representations of $G$. We often abuse notation by using the same symbol for an element of $G^{*}$, a representation in its equivalence class, and the character of such a representation. Over a field $k$ of good positive 
characteristic, we abuse notation by using $G^{*}$ instead to represent Brauer characters of $G$ over $k$.

Define the module $\Gamma_{G}$ by

$$
\Gamma_{G}=\sum_{P \in X(N)} \operatorname{Ind}_{G_{P}}^{G}\left(\sum_{\ell=1}^{e_{P}-1} \ell \theta_{P}^{\ell}\right)
$$

where $\theta_{P}$ is the ramification character at a point $P$. Thanks to Nakajima $[\mathrm{N}]$, it is known that there is a $G$-module $\tilde{\Gamma}_{G}$ such that $\left[\Gamma_{G}\right]=|G| \cdot\left[\tilde{\Gamma}_{G}\right]$ (see also $\mathrm{B})$. We call $\tilde{\Gamma}_{G}$ the ramification module.

In the case of the modular curve $X(N)$, let $\pi_{\theta_{P}}$ denote the induced representation from a character $\theta_{P}$. Then by definition,

$$
\tilde{\Gamma}_{G}=\frac{\bar{R}_{2}\left[\pi_{\theta_{1}}\right]+\bar{R}_{3}\left(\left[\pi_{\theta_{2}}\right]+2\left[\pi_{\theta_{2}^{2}}\right]\right)+\bar{R}_{N}\left(\left[\pi_{\theta_{3}}\right]+\ldots+(N-1)\left[\pi_{\theta_{3}^{N-1}}\right]\right)}{|G|}
$$

where $\bar{R}_{i}$ is the number of $P \in X(N)$ with $G_{P}=H_{i}$. Recall from section 2.1 that

$$
\bar{R}_{2}=\frac{|G|}{2}, \quad \bar{R}_{3}=\frac{|G|}{3}, \quad \bar{R}_{N}=\frac{|G|}{N}
$$

so that in fact

$$
\tilde{\Gamma}_{G}=\frac{1}{2}\left[\pi_{\theta_{1}}\right]+\frac{1}{3}\left(\left[\pi_{\theta_{2}}\right]+2\left[\pi_{\theta_{2}^{2}}\right]\right)+\frac{1}{N}\left(\left[\pi_{\theta_{3}}\right]+\ldots+(N-1)\left[\pi_{\theta_{3}^{N-1}}\right]\right) .
$$

This can easily be computed using the induced characters from section 4 To combine the results of sections 4.1 and 4.2. we must look at the congruence class of $N$ modulo 24 . We have

$$
\begin{aligned}
& N \equiv 1(\bmod 24) \Longrightarrow N \equiv 1(\bmod 4), N \equiv 1(\bmod 8), N \equiv 1(\bmod 12), \\
& N \equiv 5(\bmod 24) \Longrightarrow N \equiv 1(\bmod 4), N \equiv 5(\bmod 8), N \equiv 5(\bmod 12), \\
& N \equiv 7(\bmod 24) \Longrightarrow N \equiv 3(\bmod 4), N \equiv 7(\bmod 8), N \equiv 7(\bmod 12), \\
& N \equiv 11(\bmod 24) \Longrightarrow N \equiv 3(\bmod 4), N \equiv 3(\bmod 8), N \equiv 11(\bmod 12), \\
& N \equiv 13(\bmod 24) \Longrightarrow N \equiv 1(\bmod 4), N \equiv 5(\bmod 8), N \equiv 1(\bmod 12), \\
& N \equiv 17(\bmod 24) \Longrightarrow N \equiv 1(\bmod 4), N \equiv 1(\bmod 8), N \equiv 5(\bmod 12), \\
& N \equiv 19(\bmod 24) \Longrightarrow N \equiv 3(\bmod 4), N \equiv 3(\bmod 8), N \equiv 7(\bmod 12), \\
& N \equiv 23(\bmod 24) \Longrightarrow N \equiv 3(\bmod 4), N \equiv 7(\bmod 8), N \equiv 11(\bmod 12) . \\
& \text { Let } \mathcal{Q} \text { denote the set of quadratic residues }(\bmod N), \text { let } \mathcal{N} \text { denote the set } \\
& \text { of quadratic non-residues }(\bmod N) \text { and let }
\end{aligned}
$$




$$
S_{\mathcal{Q}}=\sum_{\ell \in \mathcal{Q}} \ell, \quad S_{\mathcal{N}}=\sum_{\ell \in \mathcal{N}} \ell=\frac{N(N-1)}{2}-S_{\mathcal{Q}} .
$$

If $N \equiv 1(\bmod 4)$, then

$$
S_{\mathcal{Q}}=S_{\mathcal{N}}=\frac{N(N-1)}{4}
$$

If $N \equiv 3(\bmod 4)$, then $S_{\mathcal{Q}}$ and $S_{\mathcal{N}}$ are not equal.

We prove the following result. An empty sum is by convention zero.

Theorem 8 We have the following decomposition of the ramification module:

- If $N \equiv 1(\bmod 24)$, let $m=\frac{13 N-13}{12}$. Then

$$
\begin{aligned}
\tilde{\Gamma}_{G}= & \frac{m}{2} W^{\prime}+\frac{m}{2} W^{\prime \prime}+m V+m \sum_{\beta} X_{\beta} \\
& +m \sum_{\alpha(i)=\alpha(\omega)=1} W_{\alpha}+(m+1) \sum_{\alpha(i)=1, \alpha(\omega) \neq 1} W_{\alpha} \\
& +(m+1) \sum_{\alpha(i) \neq 1, \alpha(\omega)=1} W_{\alpha}+(m+2) \sum_{\alpha(i) \neq 1, \alpha(\omega) \neq 1} W_{\alpha} .
\end{aligned}
$$

- If $N \equiv 5(\bmod 24)$, let $m=\frac{13 N-5}{12}$. Then

$$
\begin{aligned}
\tilde{\Gamma}_{G}= & \frac{m+1}{2} W^{\prime}+\frac{m+1}{2} W^{\prime \prime}+m V \\
& +m \sum_{\beta(\omega)=1} X_{\beta}+(m-1) \sum_{\beta(\omega) \neq 1} X_{\beta} \\
& +m \sum_{\alpha(i)=1} W_{\alpha}+(m+1) \sum_{\alpha(i) \neq 1} W_{\alpha} .
\end{aligned}
$$

- If $N \equiv 7(\bmod 24)$, let $m=\frac{13 N-7}{12}$. Then

$$
\begin{aligned}
\tilde{\Gamma}_{G}= & \left.\left.+\left(\frac{7 N-1}{24}+\frac{S_{\mathcal{Q}}}{N}\right)\right) X^{\prime}+\left(\frac{7 N-1}{24}+\frac{S_{\mathcal{N}}}{N}\right)\right) X^{\prime \prime}+m V \\
& +m \sum_{\beta(i)=1} X_{\beta}+(m-1) \sum_{\beta(i)=-1} X_{\beta} \\
& +m \sum_{\alpha(\omega)=1} W_{\alpha}+(m+1) \sum_{\alpha(\omega)=-1} W_{\alpha} .
\end{aligned}
$$


- If $N \equiv 11(\bmod 24)$, let $m=\frac{13 N+1}{12}$. Then

$$
\begin{aligned}
\tilde{\Gamma}_{G}= & \left(\frac{7 N-5}{24}+\frac{S_{\mathcal{Q}}}{N}\right) X^{\prime}+\left(\frac{7 N-5}{24}+\frac{S_{\mathcal{N}}}{N}\right) X^{\prime \prime}+m V+m \sum_{\alpha} W_{\alpha} \\
& +m \sum_{\beta(i)=\beta(\omega)=1} X_{\beta}+(m-1) \sum_{\beta(i)=1, \beta(\omega) \neq 1} X_{\beta} \\
& +(m-1) \sum_{\beta(i)=-1, \beta(\omega)=1} X_{\beta}+(m-2) \sum_{\beta(i)=-1, \beta(\omega) \neq 1} X_{\beta} .
\end{aligned}
$$

- If $N \equiv 13(\bmod 24)$, let $m=\frac{13 N-13}{12}$. Then

$$
\begin{aligned}
\tilde{\Gamma}_{G}= & \frac{m}{2} W^{\prime}+\frac{m}{2} W^{\prime}+m V+m \sum_{\beta} X_{\beta} \\
& +m \sum_{\alpha(i)=\alpha(\omega)=1} W_{\alpha}+(m+1) \sum_{\alpha(i)=1, \alpha(\omega) \neq 1} W_{\alpha} \\
& +(m+1) \sum_{\alpha(i) \neq 1, \alpha(\omega)=1} W_{\alpha}+(m+2) \sum_{\alpha(i) \neq 1, \alpha(\omega) \neq 1} W_{\alpha} .
\end{aligned}
$$

- If $N \equiv 17(\bmod 24)$, let $m=\frac{13 N-5}{12}$. Then

$$
\begin{aligned}
\tilde{\Gamma}_{G}= & \frac{m}{2} W^{\prime}+\frac{m}{2} W^{\prime \prime}+m V \\
& +m \sum_{\beta(\omega)=1} X_{\beta}+(m-1) \sum_{\beta(\omega) \neq 1} X_{\beta} \\
& +m \sum_{\alpha(i)=1} W_{\alpha}+(m+1) \sum_{\alpha(i) \neq 1} W_{\alpha} .
\end{aligned}
$$

- If $N \equiv 19(\bmod 24)$, let $m=\frac{13 N-7}{12}$. Then

$$
\begin{aligned}
\tilde{\Gamma}_{G}= & \left(\frac{7 N-13}{24}+\frac{S_{\mathcal{Q}}}{N}\right) X^{\prime}+\left(\frac{7 N-13}{24}+\frac{S_{\mathcal{N}}}{N}\right) X^{\prime \prime}+m V \\
& +m \sum_{\beta(i)=1} X_{\beta}+(m-1) \sum_{\beta(i)=-1} X_{\beta} \\
& +m \sum_{\alpha(\omega)=1} W_{\alpha}+(m+1) \sum_{\alpha(\omega)=1} W_{\alpha} .
\end{aligned}
$$


- If $N \equiv 23(\bmod 24)$, let $m=\frac{13 N+1}{12}$. Then

$$
\begin{aligned}
\tilde{\Gamma}_{G}= & \left(\frac{7 N+7}{24}+\frac{S_{\mathcal{Q}}}{N}\right) X^{\prime}+\left(\frac{7 N+7}{24}+\frac{S_{\mathcal{N}}}{N}\right) X^{\prime \prime}+m V+m \sum_{\alpha} W_{\alpha} \\
& +m \sum_{\beta(i)=\beta(\omega)=1} X_{\beta}+(m-1) \sum_{\beta(i)=1, \beta(\omega)=\neq 1} X_{\beta} \\
& +(m-1) \sum_{\beta(i)=-1, \beta(\omega)=1} X_{\beta}+(m-2) \sum_{\beta(i)=-1, \beta(\omega) \neq 1} X_{\beta} .
\end{aligned}
$$

proof: It is straightforward to verify the theorem case-by-case using (12). As an intermediate step, we tabulate the multiplicities of the irreducible representations in the induced representations which comprise $\Gamma_{G}$. These tables are included in Appendix $\$ 10$. We sketch the case $N \equiv 1(\bmod 24)$.

We begin with the multiplicity of $V$. According to (12) and the $N \equiv 1$ (mod 24) table in Appendix 10 , the multiplicity of $V$ in $\tilde{\Gamma}_{G}$. is

$$
\frac{1}{2} \frac{N-1}{2}+\frac{1}{3} N-1+\frac{1}{N} \frac{N(N-1)}{2}=\frac{13 N-13}{12}=m .
$$

The multiplicity of an $X_{\beta}$ (or a $W_{\alpha}$ with $\alpha(i)=\alpha(\omega)=1$ ) in $\tilde{\Gamma}_{G}$ is the same, since they have the same rows in the table as $V$. Next consider the multiplicities of $W^{\prime}$ and $W^{\prime \prime}$. Since $S_{\mathcal{Q}}=S_{\mathcal{N}}=\frac{N(N-1)}{4}$, these rows in the table are exactly half of the $V$ row, so the multiplicities of $W^{\prime}$ and $W^{\prime \prime}$ are both

$$
\frac{1}{2} \frac{N-1}{4}+\frac{1}{3} \frac{N-1}{2}+\frac{1}{N} \frac{N(N-1)}{4}=\frac{13 N-13}{24}=\frac{m}{2} .
$$

Next consider a $W_{\alpha}$ with $\alpha(i)=1, \alpha(\omega) \neq 1$. This row of the table differs from the $V$ row in the second column, where it is $N+2$ instead of $N-1$. Since this entry is divided by 3 in the computation, the multiplicity in $\tilde{\Gamma}_{G}$ of such a $W_{\alpha}$ will be one more than the multiplicity of $V$, or $m+1$. More directly,

$$
\frac{1}{2} \frac{N-1}{2}+\frac{1}{3} N+2+\frac{1}{N} \frac{N(N-1)}{2}=\frac{13 N-13}{12}=m+1 .
$$

Similarly, consider a $W_{\alpha}$ with $\alpha(i) \neq 1, \alpha(\omega)=1$. This row of the table differs from the $V$ row in the first column, where again the net result is to add one to the multiplicity. In this case,

$$
\frac{1}{2} \frac{N+3}{2}+\frac{1}{3} N-1+\frac{1}{N} \frac{N(N-1)}{2}=\frac{13 N-13}{12}=m+1 .
$$


as desired. Finally, consider a $W_{\alpha}$ with $\alpha(i) \neq 1, \alpha(\omega) \neq 1$. We see that both of the first two columns of the table differ in this row from the $V$ row, for a net addition of 2 to the multiplicity. In this case,

$$
\frac{1}{2} \frac{N+3}{2}+\frac{1}{3} N+2+\frac{1}{N} \frac{N(N-1)}{2}=\frac{13 N-13}{12}=m+2 .
$$

This completes the verification of the theorem in the case $N \equiv 1(\bmod 24)$. A similar analysis can be used on the other cases. $\square$

If $\tilde{\Gamma}_{G}$ has a $\mathbb{Q}[G]$-module structure, it may be computed more simply JK]. In this case, the formula for it is

$$
\tilde{\Gamma}_{G}=\bigoplus_{\pi \in G^{*}}\left[\sum_{\ell=1}^{L}\left(\operatorname{dim} \pi-\operatorname{dim}\left(\pi^{H_{\ell}}\right)\right) \frac{R_{\ell}}{2}\right] \pi
$$

where $\left\{H_{1}, \ldots, H_{L}\right\}$ represent the set of conjugacy classes of cyclic subgroups of $G$ ). If $\tilde{\Gamma}_{G}$ is not $\mathcal{G}=\operatorname{Gal}(\mathbb{Q}(G) / \mathbb{Q})$-invariant, so there is no $\mathbb{Q}[G]$-module structure, then

$$
\bigoplus_{\pi \in G^{*}}|\mathcal{G}| \cdot\left[\sum_{\ell=1}^{L}\left(\operatorname{dim} \pi-\operatorname{dim}\left(\pi^{H_{\ell}}\right)\right) \frac{R_{\ell}}{2}\right] \pi
$$

yields the $G$-module decomposition of the Galois-closure of $\tilde{\Gamma}_{G}$ (i.e., the smallest $\mathcal{G}$-invariant $G$-module containing $\tilde{\Gamma}_{G}$ ). See the remark after Corollary 6 in [JK]. Also, compare this with Lemma 3 in [KP (we thank Dipendra Prasad for providing us with this reference).

This motivates the following, as stated in the introduction.

Theorem 9 For $N>5$ prime, the ramification module of $X(N)$ over $X(1)$ is $\mathcal{G}$-invariant if and only if $N \equiv 1(\bmod 4)$.

proof: . As noted in 93 , the action of $\mathcal{G}$ is as follows:

- On $X^{\prime}$ and $X^{\prime \prime}$ or $W^{\prime}$ and $W^{\prime \prime}$ : The induced characters from $H_{1}$ and $\mathrm{H}_{2}$ are invariant under this action. The induced characters from $\mathrm{H}_{3}$ are not; the action exchanges $\theta^{k}$ where $k$ is a quadratic residue $(\bmod N)$ with $\theta^{k^{\prime}}$ where $k^{\prime}$ is not a quadratic residue $(\bmod N)$. However the sum appearing in the ramification module

$$
\operatorname{Ind}_{G_{P}}^{G}\left(\sum_{\ell=1}^{e_{P}-1} \ell \theta_{P}^{\ell}\right)=\sum_{\ell=1}^{N-1} \ell \operatorname{Ind}_{H_{3}}^{G} \theta^{\ell}
$$


will include

$$
\sum_{\left(\frac{\ell}{N}\right)=1} \ell W^{\prime}+\sum_{\left(\frac{\ell}{N}\right)=-1} \ell W^{\prime \prime}
$$

if $N \equiv 1(\bmod 4)$, or

$$
\sum_{\left(\frac{\ell}{N}\right)=1} \ell X^{\prime}+\sum_{\left(\frac{\ell}{N}\right)=-1} \ell X^{\prime \prime}
$$

if $N \equiv 3(\bmod 4)$. Here $\left(\frac{*}{N}\right)$ denotes the Legendre symbol. If $N \equiv 1$ $(\bmod 4)$, then the sum of the quadratic residues is the same as the sum of the nonresidues, so the multiplicities of $W^{\prime}$ and $W^{\prime \prime}$ are the same. If $N \equiv 3(\bmod 4)$, then the sum of the quadratic residues is not the same as the sum of the quadratic nonresidues, so these multiplicities are different. Therefore this part of the ramification module module is Galois invariant if and only if $N \equiv 1(\bmod 4)$.

- On $X_{\beta}$ : The multiplicity of a discrete series representation $X_{\beta}$ depends only on $\beta(i)$ and $\beta(\omega)$. Observe that $\mathcal{G}$ fixes elements of $\mathbb{Q}$, therefore cannot exchange $X_{\beta}$ having $\beta(i)=1$ with an $X_{\beta}$ having $\beta(i)=-1$, or $X_{\beta}$ having $\beta(\omega)=1$ with $X_{\beta}$ having $\beta(\omega) \neq 1$. Therefore this part of the ramification module is Galois invariant.

- $O n W_{\alpha}$ : Similarly, the multiplicity of a principal series representation $W_{\alpha}$ depends only on $\alpha(i)$ and $\alpha(\omega)$. Again, $\mathcal{G}$ will not exchange these, so this part of the module is Galois invariant.

Thus we see that $\tilde{\Gamma}_{G}$ is Galois-invariant if and only if $N \equiv 1(\bmod 4)$.

In this case, we can use formula (14) to compute the ramification module directly from the restricted representations in section 4 , and get the same result with fewer steps. If $N \equiv 3(\bmod 4)$, formula (15) will yield the Galois closure of the ramification module.

\section{Equivariant degree and Riemann-Roch space}

Now we will define and compute the equivariant degree of a $G$-invariant divisor. This, together with Borne's formula (11) will allow us to compute the $G$-module structure of the Riemann-Roch space $L(D)$. 
Fix a point $P \in X(N)$ and let $D$ be a divisor on $X(N)$ of the form

$$
D=\frac{r}{e_{P}} \sum_{g \in G} g(P)=r \sum_{g \in G / G_{P}} g(P),
$$

where $G_{P}$ denotes the stabilizer in $G$ of $P$ and $e_{P}=\left|G_{P}\right|$ denotes the ramification index at $P$.

When $r=1$ such a divisor is called a reduced orbit by Borne $[\mathrm{B}$. When $r$ is a multiple of $e_{P}$ then $D$ is the pull-back of a divisor on $X(1)$ via $\psi_{N}$ in (3i).

When $D$ is as above, the equivariant degree of $D$ is the virtual representation

$$
\operatorname{deg}_{e q}(D)=\operatorname{Ind}_{G_{P}}^{G}\left(\sum_{\ell=1}^{r} \theta_{P}^{-\ell}\right),
$$

where $\theta_{P}$ is the ramification character of $X(N)$ at $P$ (a character of $G_{P}$ ). In general, the equivariant degree is additive on disjointly supported divisors. See for example Borne [B] for more details.

Example 10 Consider a hyperelliptic curve $X$ for which every ramification point $P$ has $e_{P}=2$. Let $D$ denote the sum of all the ramification points of $X$. Then (by definition) $\operatorname{deg}_{e q}(D)=\Gamma_{G}=2 \tilde{\Gamma}_{G}$.

On the modular curve $X(N)$, the results of section 2.1 tell us that there are only four types of reduced orbits to consider: the stabilizer $G_{P}$ of a point $P$ in the support of $D$ may have order $1,2,3$, or $N$. Let $D_{0}, D_{1}, D_{2}$, and $D_{3}$ denote reduced orbits of each type. We compute the equivariant degree of $r D_{i}$ in each case.

Case $1: G_{P}$ is trivial. If $G_{P}$ has order 1 , then the ramification character is trivial. $D_{0}$ is an entire orbit, in fact the pullback of a divisor on $X(1)$. The equivariant degree of $r D_{0}$ is $r \cdot k[G]$, a multiple of the regular representation $k[G]$ of $G$.

Case 2: $G_{P} \cong H_{1}$. If $G_{P}$ has order 2 , then the equivariant degree of $D_{1}$ is $\operatorname{Ind}_{G_{P}}^{G} \theta_{1}$ (which was computed in section 4.1). Even multiples of $D_{1}$ will be pullbacks from $X(1)$, so $\operatorname{deg}_{e q}\left(2 r D_{1}\right)=r \cdot k[G]$, and $\operatorname{deg}_{e q}\left((2 r+1) D_{1}\right)=r \cdot k[G]+\operatorname{Ind}_{G_{P}}^{G} \theta_{1}$. 
Case 3: $G_{P} \cong H_{2}$. The equivariant degree of $D_{2}$ is $\operatorname{Ind}_{G_{P}}^{G} \theta_{2}^{2}$, which was computed in section 4.2. Note that $\operatorname{Ind}_{G_{P}}^{G} \theta_{2}^{2}=\operatorname{Ind}_{G_{P}}^{G} \theta_{2}$, so $\operatorname{deg}_{e q}\left(2 D_{2}\right)=$ $2 \operatorname{Ind}_{G_{P}}^{G} \theta_{2}$. But $3 D_{2}$ is a pullback from $X(1)$, so $\operatorname{deg}_{e q}\left(3 D_{2}\right)=k[G]$, and more generally $\operatorname{deg}_{\text {eq }}\left(3 r D_{2}\right)=r \cdot k[G], \operatorname{deg}_{e q}\left((3 r+1) D_{2}\right)=r \cdot k[G]+$ $\operatorname{Ind}_{G_{P}}^{G} \theta_{2}$, and $\operatorname{deg}_{e q}\left((3 r+2) D_{2}\right)=r \cdot k[G]+2 \operatorname{Ind}_{G_{P}}^{G} \theta_{2}$.

Case $4: G_{P} \cong H_{3}$. This case is more complicated because $\operatorname{Ind}_{H_{3}}^{G} \theta_{3}^{k}$ depends on whether $k$ is a quadratic residue modulo $N$ (see section [4.3). If $1 \leq r \leq N-1$, let $q_{r}$ denote the number of quadratic residues modulo $N$ in the set $N-r, \ldots, N-1$, and let $n_{r}$ denote the number of quadratic nonresidues modulo $N$ in the same set. Then the equivariant degree $\operatorname{deg}_{e q}\left(r D_{3}\right)$ will be

$$
\operatorname{deg}_{e q}\left(r D_{3}\right)=r\left(\sum_{\beta} X_{\beta}+V+\sum_{\alpha} W_{\alpha}\right)+\left\{\begin{array}{lll}
q_{r} W^{\prime}+n_{r} W^{\prime \prime}, & N \equiv 1 & (\bmod 4) \\
q_{r} X^{\prime}+n_{r} X^{\prime \prime}, & N \equiv 3 & (\bmod 4) .
\end{array}\right.
$$

Now we would like to compute the $G$-module structure of the RiemannRoch space $L(D)$ for a non-special $G$-invariant divisor $D$. First, let us consider which $G$-invariant divisors are non-special. To be non-special, it is sufficient to have $\operatorname{deg} D>2 g-2$, where

$$
g=1+\frac{(N-6)\left(N^{2}-1\right)}{24}
$$

is the genus of $X(N)$, so

$$
2 g-2=\frac{(N-6)\left(N^{2}-1\right)}{12}=\frac{(N-6)}{6 N}|G| .
$$

The reduced orbits $D_{0}, D_{1}$ and $D_{2}$ have degrees $|G|,|G| / 2$ and $|G| / 3$, respectively, which are greater than $2 g-2$ for any $N$, so these are always nonspecial. However, the reduced orbit $D_{3}$ has degree $|G| / N$, which is greater than $2 g-2$ only if $N<12$. A multiple of this reduced orbit, $r D_{3}$ will be non-special if $r>(N-6) / 6$.

Once the equivariant degree of a non-special divisor has been computed, it is simple to combine this with the ramification module, using Borne's formula, to find the $G$-module structure of the Riemann-Roch space $L(D)$. The number of cases becomes quite cumbersome, but in each case there will 
be a number $M$ such that all but the two smallest nontrivial irreducibles (either $W^{\prime}$ and $W^{\prime \prime}$ or $X^{\prime}$ and $X^{\prime \prime}$ ) will have multiplicity either $M, M+1$, $M+2$, or $M+3$, and the multiplicities of the two smallest irreducibles will be half of one of these numbers.

There is one special case worth mentioning: if $D$ is a pull-back of an effective divisor $\bar{D}$ on $X(1)$ via $\psi_{N}$, then it will be non-special; moreover in this case

$$
\left[\operatorname{deg}_{e q}(D)\right]=\operatorname{deg}\left(D_{0}\right)[k[G]]
$$

and we will have the following decomposition of the Riemann-Roch space

$$
[L(D)]=(1+\operatorname{deg}(\bar{D}))[k[G]]-\left[\tilde{\Gamma}_{G}\right]
$$

where $\tilde{\Gamma}_{G}$ is as above.

\section{$7 \quad$ Examples}

The computer algebra system GAP computes information about $P S L(2, N)$; one can use it to compute character tables, induced characters and Schur inner products. In the examples of $X(7)$ and $X(11)$ below, we use GAP to explicitly compute the $G$-module structure of the ramification module and some Riemann-Roch spaces, in the cases $N=7$ and $N=11$. Section [1] at the end of this paper gives computations of the ramification module for higher $N$, and the GAP programs we used.

\subsection{The case $N=7$}

The equivalence classes of irreducible representations of $P S L(2,7)$ are $G^{*}=$ $\left\{\pi_{1}, \pi_{2}, \ldots, \pi_{6}\right\}$, where

$\operatorname{dim}\left(\pi_{1}\right)=1, \quad \operatorname{dim}\left(\pi_{2}\right)=\operatorname{dim}\left(\pi_{3}\right)=3, \quad \operatorname{dim}\left(\pi_{4}\right)=6, \quad \operatorname{dim}\left(\pi_{5}\right)=7, \quad \operatorname{dim}\left(\pi_{6}\right)=8$.

We can identify

$$
\pi_{1}=\mathbf{1}_{G}, \quad \pi_{2}=X^{\prime}, \quad \pi_{3}=X^{\prime \prime}, \quad \pi_{4}=X_{\beta}, \quad \pi_{5}=V, \quad \pi_{5}=W_{\alpha} .
$$

Let $\zeta=e^{\frac{2 \pi i}{7}}$ and let $\mathbb{Q}(q)$ denote the (quadratic) extension of $\mathbb{Q}$ by $q=\zeta+\zeta^{2}+\zeta^{4}$. Let $\mathcal{G}$ denote the Galois group of $\mathbb{Q}(q) / \mathbb{Q}$. Then $\mathcal{G}$ acts 
on the irreducible representations $G^{*}$ by swapping the two 3-dimensional representations (i.e., $\pi_{2}=X^{\prime}$ and $\pi_{3}=X^{\prime \prime}$ ) and fixing the others.

There are 4 conjugacy classes of non-trivial cyclic subgroups of $G$, whose representatives are denoted by $H_{1}$ (order 2), $H_{2}$ (order 3 ), $H_{3}$ (order 7), $H_{4}$ (order 4). Thus $H_{4}$ is spurious. We use GAP to compute the induced characters:

- If $\theta_{1} \in H_{1}^{*}$ then $\pi_{\theta_{1}}=\operatorname{In} d_{H_{1}}^{G} \theta_{1}$ is 84-dimensional. Moreover,

$$
\pi_{\theta_{1}} \cong \begin{cases}2 \pi_{2} \oplus 2 \pi_{3} \oplus 2 \pi_{4} \oplus 4 \pi_{5} \oplus 4 \pi_{6}, & \theta_{1} \neq 1, \\ \pi_{1} \oplus \pi_{2} \oplus \pi_{3} \oplus 4 \pi_{4} \oplus 3 \pi_{5} \oplus 4 \pi_{6}, & \theta_{1}=1 .\end{cases}
$$

- If $\theta_{2} \in H_{2}^{*}$ then $\pi_{\theta_{2}}=\operatorname{Ind} d_{H_{2}}^{G} \theta_{2}$ is 56-dimensional. Moreover,

$$
\pi_{\theta_{2}} \cong \begin{cases}\pi_{2} \oplus \pi_{3} \oplus 2 \pi_{4} \oplus 2 \pi_{5} \oplus 3 \pi_{6}, & \theta_{2} \neq 1, \\ \pi_{1} \oplus \pi_{2} \oplus \pi_{3} \oplus 2 \pi_{4} \oplus 3 \pi_{5} \oplus 2 \pi_{6}, & \theta_{2}=1 .\end{cases}
$$

- If $\theta_{3} \in H_{3}^{*}$ is a fixed non-trivial character then $\pi_{\theta_{3}}=\operatorname{Ind}_{H_{3}}^{G} \theta_{3}$ is 24dimensional. Moreover,

$$
\pi_{\theta_{3}^{k}} \cong \begin{cases}\pi_{3} \oplus \pi_{4} \oplus \pi_{5} \oplus \pi_{6} & k \text { quad. non-res. } \quad(\bmod 7) \\ \pi_{2} \oplus \pi_{4} \oplus \pi_{5} \oplus \pi_{6}, & k \text { quad. res. } \quad(\bmod 7), \\ \pi_{1} \oplus \pi_{5} \oplus 2 \pi_{6}, & k \equiv 0 \quad(\bmod 7) .\end{cases}
$$

These computations agree with the computations in section 4. This data allows us to easily compute the ramification module using equation (12):

$$
\left[\tilde{\Gamma}_{G}\right]=\left[3 \pi_{2} \oplus 4 \pi_{3} \oplus 6 \pi_{4} \oplus 7 \pi_{5} \oplus 8 \pi_{6}\right] .
$$

Note that this is not Galois-invariant, because $\pi_{2}$ and $\pi_{3}$ have different multiplicities. A naïve computation of the ramification module, using (14), yields the following. For brevity, we represent $m_{1}\left[\pi_{1}\right]+\ldots+m_{6}\left[\pi_{6}\right]$ as $\left(m_{1}, \ldots, m_{6}\right)$. We compute, using GAP, the quantities

$$
\begin{aligned}
& \left(\operatorname{dim} \pi-\operatorname{dim}\left(\pi^{H_{1}}\right)\right)_{i=1 . .6}=(1,3,3,6,7,8)-(1,1,1,4,3,4)=(0,2,2,2,4,4), \\
& \left(\operatorname{dim} \pi-\operatorname{dim}\left(\pi^{H_{2}}\right)\right)_{i=1 . .6}=(1,3,3,6,7,8)-(1,1,1,2,3,2)=(0,2,2,4,4,6),
\end{aligned}
$$


$\left(\operatorname{dim} \pi-\operatorname{dim}\left(\pi^{H_{3}}\right)\right)_{i=1 . .6}=(1,3,3,6,7,8)-(1,0,0,0,1,2)=(0,3,3,6,6,6)$.

$\left(\operatorname{dim} \pi-\operatorname{dim}\left(\pi^{H_{4}}\right)\right)_{i=1 . .6}=(1,3,3,6,7,8)-(1,1,1,2,1,2)=(0,2,2,4,6,6)$.

Combining this with $R_{1}=R_{2}=R_{3}=1$ and $R_{4}=0$ in (12) gives

$$
\begin{gathered}
{\left[\tilde{\Gamma}_{G}\right]=\left[\bigoplus_{i=1}^{6}\left[\sum_{\ell=1}^{4}\left(\operatorname{dim} \pi_{i}-\operatorname{dim}\left(\pi_{i}^{H_{\ell}}\right)\right) \frac{R_{\ell}}{2}\right] \pi_{i}\right]} \\
=(0,2,2,2,4,4) \frac{1}{2}+(0,2,2,4,4,6) \frac{1}{2}+(0,3,3,6,6,6) \frac{1}{2}+(0,2,2,4,6,6) \frac{0}{2} \\
=(0,7 / 2,7 / 2,6,7,8) \\
=\frac{7}{2}\left[\pi_{2}\right]+\frac{7}{2}\left[\pi_{3}\right]+6\left[\pi_{4}\right]+7\left[\pi_{5}\right]+8\left[\pi_{6}\right] .
\end{gathered}
$$

This is impossible, and therefore we see that $\tilde{\Gamma}_{G}$ does not have a $\mathbb{Q}[G]$ module structure in this case. However, the result does agree with (15): the multiplicity given for $\pi_{2}$ and $\pi_{3}$ is the average of the two actual multiplicities.

Now we will use GAP to compute the equivariant degree and RiemannRoch module for some example divisors. As noted in section 6, any effective $G$-invariant divisor on $X(7)$ will be non-special. Since $X(1)$ is genus zero, for $N=7$, Borne's formula (II) becomes

$$
\begin{aligned}
{[L(D)]=} & {\left[\pi_{1} \oplus 3 \pi_{2} \oplus 3 \pi_{3} \oplus 6 \pi_{4} \oplus 7 \pi_{5} \oplus 8 \pi_{6}\right]+\left[\operatorname{deg}_{e q}(D)\right]-\left[\tilde{\Gamma}_{G}\right] } \\
= & {\left[\pi_{1} \oplus 3 \pi_{2} \oplus 3 \pi_{3} \oplus 6 \pi_{4} \oplus 7 \pi_{5} \oplus 8 \pi_{6}\right]+\left[\operatorname{deg}_{e q}(D)\right] } \\
& -3\left[\pi_{2}\right]-4\left[\pi_{3}\right]-6\left[\pi_{4}\right]-7\left[\pi_{5}\right]-8\left[\pi_{6}\right] \\
= & {\left[\pi_{1}\right]-\left[\pi_{3}\right]+\left[\operatorname{deg}_{e q}(D)\right] . }
\end{aligned}
$$

If $D_{1}$ is the reduced orbit of a point with stabilizer $H_{1}$, then

$$
\left[\operatorname{deg}_{e q}\left(D_{1}\right)\right]=\left[\pi_{\theta_{1}}\right]=\left[2 \pi_{2} \oplus 2 \pi_{3} \oplus 2 \pi_{4} \oplus 4 \pi_{5} \oplus 4 \pi_{6}\right]
$$

and

$$
\left[L\left(D_{1}\right)\right]=\left[\pi_{1} \oplus 2 \pi_{2} \oplus \pi_{3} \oplus 2 \pi_{4} \oplus 4 \pi_{5} \oplus 4 \pi_{6}\right] .
$$

If $D_{2}$ is the reduced orbit of a point with stabilizer $\mathrm{H}_{2}$, then

$$
\left[\operatorname{deg}_{e q}\left(D_{2}\right)\right]=\left[\pi_{\theta_{2}}\right]=\left[\pi_{2} \oplus \pi_{3} \oplus 2 \pi_{4} \oplus 2 \pi_{5} \oplus 3 \pi_{6}\right]
$$




$$
\left[\operatorname{deg}_{e q}\left(2 D_{2}\right)\right]=\left[2 \pi_{\theta_{2}}\right]=\left[2 \pi_{2} \oplus 2 \pi_{3} \oplus 4 \pi_{4} \oplus 4 \pi_{5} \oplus 6 \pi_{6}\right]
$$

and

$$
\begin{gathered}
{\left[L\left(D_{2}\right)\right]=\left[\pi_{1} \oplus \pi_{2} \oplus 2 \pi_{4} \oplus 2 \pi_{5} \oplus 3 \pi_{6}\right],} \\
{\left[L\left(2 D_{2}\right)\right]=\left[\pi_{1} \oplus 2 \pi_{2} \oplus \pi_{3} \oplus 4 \pi_{4} \oplus 4 \pi_{5} \oplus 6 \pi_{6}\right] .}
\end{gathered}
$$

If $D_{3}$ is the reduced orbit of a point with stabilizer $H_{3}$, then

$$
\begin{gathered}
{\left[\operatorname{deg}_{e q}\left(D_{3}\right)\right]=\left[\pi_{\theta_{3}^{N-1}}\right]=\left[\pi_{3} \oplus \pi_{4} \oplus \pi_{5} \oplus \pi_{6}\right],} \\
{\left[\operatorname{deg}_{e q}\left(2 D_{3}\right)\right]=2\left[\pi_{3} \oplus \pi_{4} \oplus \pi_{5} \oplus \pi_{6}\right],} \\
{\left[\operatorname{deg}_{e q}\left(3 D_{3}\right)\right]=\left[\pi_{2}+2 \pi_{3}\right]+3\left[\pi_{4} \oplus \pi_{5} \oplus \pi_{6}\right],} \\
{\left[\operatorname{deg}_{e q}\left(4 D_{3}\right)\right]=\left[\pi_{2}+3 \pi_{3}\right]+4\left[\pi_{4} \oplus \pi_{5} \oplus \pi_{6}\right],} \\
{\left[\operatorname{deg}_{e q}\left(5 D_{3}\right)\right]=\left[2 \pi_{2}+3 \pi_{3}\right]+5\left[\pi_{4} \oplus \pi_{5} \oplus \pi_{6}\right],} \\
{\left[\operatorname{deg}_{e q}\left(6 D_{3}\right)\right]=\left[3 \pi_{2}+3 \pi_{3}\right]+6\left[\pi_{4} \oplus \pi_{5} \oplus \pi_{6}\right] .}
\end{gathered}
$$

It follows that

$$
L\left(D_{3}\right)=\left[\pi_{1}\right]-\left[\pi_{3}\right]+\left[\pi_{3} \oplus \pi_{4} \oplus \pi_{5} \oplus \pi_{6}\right]=\left[\pi_{1} \oplus \pi_{4} \oplus \pi_{5} \oplus \pi_{6}\right],
$$

which is dimension 22 , and

$$
L\left(2 D_{3}\right)=\left[\pi_{1}\right]-\left[\pi_{3}\right]+2\left[\pi_{3} \oplus \pi_{4} \oplus \pi_{5} \oplus \pi_{6}\right]=\left[\pi_{1} \oplus \pi_{3}\right]+2\left[\pi_{4} \oplus \pi_{5} \oplus \pi_{6}\right],
$$

which is dimension 46 .

\subsection{The case $N=11$}

We next consider the case of $N=11$. Up to equivalence, the irreducible representations of $G=P S L(2,11)$ are $G^{*}=\left\{\pi_{1}, \pi_{2}, \ldots, \pi_{8}\right\}$, where

$$
\begin{gathered}
\operatorname{dim}\left(\pi_{1}\right)=1, \quad \operatorname{dim}\left(\pi_{2}\right)=\operatorname{dim}\left(\pi_{3}\right)=5, \quad \operatorname{dim}\left(\pi_{4}\right)=\operatorname{dim}\left(\pi_{5}\right)=10, \\
\operatorname{dim}\left(\pi_{6}\right)=11, \quad \operatorname{dim}\left(\pi_{7}\right)=\operatorname{dim}\left(\pi_{8}\right)=12 .
\end{gathered}
$$

and we can identify

$$
\pi_{1}=\mathbf{1}_{G}, \quad \pi_{2}=X^{\prime}, \quad \pi_{3}=X^{\prime \prime}, \quad \pi_{4}=X_{\beta_{1}}, \quad \pi_{5}=X_{\beta_{2}},
$$




$$
\pi_{6}=V, \quad \pi_{7}=W_{\alpha_{1}}, \quad \pi_{8}=W_{\alpha_{2}} .
$$

We can use GAP to compute the character table of $G$ and from the character values we can deduce some things about the characters $\alpha_{1}, \alpha_{2}: \mathbb{F}^{\times} \rightarrow \mathbb{C}^{\times}$and $\beta_{1}, \beta_{2}: T \rightarrow \mathbb{C}^{\times}$. The generator $\varepsilon$ of $\mathbb{F}^{\times}$has order 10 ; it is sent by $\alpha_{1}$ to $e^{4 \pi i / 5}$ and by $\alpha_{2}$ to $e^{2 \pi i / 5}$. The generator $\tau$ of $T$ has order 12 ; it is sent by $\beta_{1}$ to $e^{2 \pi i / 3}$ and by $\beta_{2}$ to $e^{\pi i / 3}$. Because $N \equiv-1(\bmod 12)$, the numbers " $i "=\tau^{3}$ and " $\omega$ " $=\tau^{2}$ are both in $T$; we see from this that $\beta_{1}(i)=1, \beta_{2}(i)=-1$, $\beta_{1}(\omega) \neq 1$, and $\beta_{2}(\omega) \neq 1$.

Thanks to the above discussion, it should be clear that if $K=\mathbb{Q}(G)$ denotes the abelian extension of $\mathbb{Q}$ generated by the character values of $G$ then $\mathcal{G}=\operatorname{Gal}(K / \mathbb{Q})$ acts by swapping the two irreducible 5-dimensionals $X^{\prime}$ and $X^{\prime \prime}$, and the two irreducible 12-dimensionals $W_{\alpha_{1}}$ and $W_{\alpha_{2}}$, but not the two irreducible 10-dimensionals $X_{\beta_{1}}$ and $X_{\beta_{2}}$.

There are 5 conjugacy classes of cyclic subgroups of $G$, whose representatives are denoted by $H_{1}, \ldots, H_{5}$. They satisfy $\left|H_{1}\right|=2,\left|H_{2}\right|=3,\left|H_{3}\right|=11$, $\left|H_{4}\right|=5,\left|H_{5}\right|=6$. We are interested in $H_{1}, H_{2}$, and $H_{3} ; H_{4}$ and $H_{5}$ are spurious. We use GAP to compute the induced characters:

- If $\theta_{1} \in H_{1}^{*}$ then $\pi_{\theta_{1}}=\operatorname{Ind} d_{H_{1}}^{G} \theta_{1}$ is 330-dimensional. Moreover,

$$
\pi_{\theta_{1}} \cong \begin{cases}2 \pi_{2} \oplus 2 \pi_{3} \oplus 6 \pi_{4} \oplus 4 \pi_{5} \oplus 6 \pi_{6} \oplus 6 \pi_{7} \oplus 6 \pi_{8}, & \theta_{1} \neq 1 \\ \pi_{1} \oplus 3 \pi_{2} \oplus 3 \pi_{3} \oplus 4 \pi_{4} \oplus 6 \pi_{5} \oplus 5 \pi_{6} \oplus 6 \pi_{7} \oplus 6 \pi_{8}, & \theta_{1}=1\end{cases}
$$

- If $\theta_{2} \in H_{2}^{*}$ then $\pi_{\theta_{2}}=\operatorname{Ind}_{H_{2}}^{G} \theta_{2}$ is 220-dimensional. Moreover,

$$
\pi_{\theta_{2}}= \begin{cases}2 \pi_{2} \oplus 2 \pi_{3} \oplus 3 \pi_{4} \oplus 3 \pi_{5} \oplus 4 \pi_{6} \oplus 4 \pi_{7} \oplus 4 \pi_{8}, & \theta_{2} \neq 1 \\ \pi_{1} \oplus \pi_{2} \oplus \pi_{3} \oplus 4 \pi_{4} \oplus 4 \pi_{5} \oplus 3 \pi_{6} \oplus 4 \pi_{7} \oplus 4 \pi_{8}, & \theta_{2}=1 .\end{cases}
$$

- If $\theta_{3} \in H_{3}^{*}$ is a fixed non-trivial character then $\pi_{\theta_{3}}=\operatorname{Ind}_{H_{3}}^{G} \theta_{3}$ is 66dimensional. Note that this is not $\mathcal{G}$-invariant. Moreover,

$$
\pi_{\theta_{3}^{k}}= \begin{cases}\pi_{2} \oplus \pi_{4} \oplus \pi_{5} \oplus \pi_{6} \oplus \pi_{7} \oplus \pi_{8}, \quad k \text { quad. non-res. } \quad(\bmod 11), \\ \pi_{3} \oplus \pi_{4} \oplus \pi_{5} \oplus \pi_{6} \oplus \pi_{7} \oplus \pi_{8}, & k \text { quad. res. } \quad(\bmod 11), \\ \pi_{1} \oplus \pi_{6} \oplus 2 \pi_{7} \oplus 2 \pi_{8}, & k \equiv 0 \quad(\bmod 11) .\end{cases}
$$


From this data, we can easily compute the ramification module, using equation (12). The equivalence class $\left[\Gamma_{G}\right]$ equals

$$
\begin{gathered}
\bar{R}_{2}\left[\pi_{\theta_{1}}\right]+\bar{R}_{3}\left(\left[\pi_{\theta_{2}}\right]+2\left[\pi_{\theta_{2}^{2}}\right]\right) \\
+\bar{R}_{N}\left(\left[\pi_{\theta_{3}}\right]+\ldots+(N-1)\left[\pi_{\theta_{3}^{N-1}}\right]\right)
\end{gathered}
$$

where $N=11$. As was mentioned above, using $\left[\mathrm{S}\right.$, we have $\bar{R}_{2}=660 / 2$, $\bar{R}_{3}=660 / 3, \bar{R}_{11}=660 / 11$. Therefore,

$$
\begin{aligned}
{\left[\Gamma_{G}\right]=} & 660 \cdot\left[\frac{1}{2} \cdot(0,2,2,6,4,6,6,6)+(0,2,2,3,3,4,4,4)\right. \\
& +\cdot(0,3,2,5,5,5,5,5)] \\
= & 660 \cdot(0,6,5,11,10,12,12,12),
\end{aligned}
$$

by the formulas for the induced characters above. Therefore, $\left[\tilde{\Gamma}_{G}\right]=(0,6,5,11,10,12,12,12)$.

As in the case of $X(7)$, this is not Galois invariant, because $\pi_{2}$ and $\pi_{3}$ have different multiplicities. Again we compute the Galois closure using equation (15):

$$
\begin{aligned}
\left(\operatorname{dim} \pi-\operatorname{dim}\left(\pi^{H_{1}}\right)\right)_{i=1 . .8} & =(1,5,5,10,10,11,12,12)-(1,3,3,4,6,5,6,6) \\
& =(0,2,2,6,4,6,6,6), \\
\left(\operatorname{dim} \pi-\operatorname{dim}\left(\pi^{H_{2}}\right)\right)_{i=1 . .8} & =(1,5,5,10,10,11,12,12)-(1,1,1,4,4,3,4,4) \\
& =(0,4,4,6,6,8,8,8), \\
\left(\operatorname{dim} \pi-\operatorname{dim}\left(\pi^{H_{3}}\right)\right)_{i=1 . .8} & =(1,5,5,10,10,11,12,12)-(1,0,0,0,0,1,2,2) \\
& =(0,5,5,10,10,10,10,10),
\end{aligned}
$$

These calculations, combined with $R_{1}=R_{2}=R_{3}=1$ and $R_{4}=R_{5}=0$, give

$$
\begin{gathered}
{\left[\tilde{\Gamma}_{G}\right]=\left[\bigoplus_{i=1}^{8}\left[\sum_{\ell=1}^{5}\left(\operatorname{dim} \pi_{i}-\operatorname{dim}\left(\pi_{i}^{H_{\ell}}\right)\right) \frac{R_{\ell}}{2}\right] \pi_{i}\right]} \\
=(0,2,2,6,4,6,6,6) \frac{1}{2}+(0,2,2,3,3,4,4,4) \frac{1}{2}+(0,5,5,10,10,10,10,10) \frac{1}{2} \\
=(0,11 / 2,11 / 2,11,10,12,12,12) \\
=\frac{11}{2}\left[\pi_{2}\right]+\frac{11}{2}\left[\pi_{3}\right]+11\left[\pi_{4}\right]+10\left[\pi_{5}\right]+12\left[\pi_{6}\right]+12\left[\pi_{7}\right]+12\left[\pi_{8}\right] .
\end{gathered}
$$


We can see directly from this calculation $\tilde{\Gamma}_{G}$ does not have a $\mathbb{Q}[G]$-module structure in this case. However, the result does agree with (15): we have computed the average of the multiplicities of the representations under the Galois action.

For every non-special $G$-equivariant divisor $D$ on $X(11)$, the formula (11) says

$$
\begin{aligned}
{[L(D)]=} & {\left[\pi_{1} \oplus 5 \pi_{2} \oplus 5 \pi_{3} \oplus 10 \pi_{4} \oplus 10 \pi_{5} \oplus 11 \pi_{6} \oplus 12 \pi_{7} \oplus 12 \pi_{8}\right] } \\
& \quad+\left[\operatorname{deg}_{e q}(D)\right]-\left[\tilde{\Gamma}_{G}\right] \\
= & {\left[\pi_{1}\right]-\left[\pi_{2}\right]-\left[\pi_{4}\right]-\left[\pi_{6}\right]+\left[\operatorname{deg}_{e q}(D)\right] . }
\end{aligned}
$$

If $D_{1}$ is the reduced orbit of a point with stabilizer $H_{1}$, then $D_{1}$ is nonspecial and

$$
\left[\operatorname{deg}_{e q}\left(D_{1}\right)\right]=\left[\pi_{\theta_{1}}\right]=\left[2 \pi_{2} \oplus 2 \pi_{3} \oplus 6 \pi_{4} \oplus 4 \pi_{5} \oplus 6 \pi_{6} \oplus 6 \pi_{7} \oplus 6 \pi_{8}\right],
$$

and

$$
\left[L\left(D_{1}\right)\right]=\left[\pi_{1} \oplus \pi_{2} \oplus 2 \pi_{3} \oplus 5 \pi_{4} \oplus 4 \pi_{5} \oplus 5 \pi_{6} \oplus 6 \pi_{7} \oplus 6 \pi_{8}\right] .
$$

If $D_{2}$ is the reduced orbit of a point with stabilizer $H_{2}$, then $D_{2}$ is non-special and

$$
\left[\operatorname{deg}_{e q}\left(D_{2}\right)\right]=\left[\pi_{\theta_{2}^{2}}\right]=\left[2 \pi_{2} \oplus 2 \pi_{3} \oplus 3 \pi_{4} \oplus 3 \pi_{5} \oplus 4 \pi_{6} \oplus 4 \pi_{7} \oplus 4 \pi_{8}\right],
$$

and

$$
\left[L\left(D_{2}\right)\right]=\left[\pi_{1} \oplus \pi_{2} \oplus 2 \pi_{3} \oplus 2 \pi_{4} \oplus 3 \pi_{5} \oplus 3 \pi_{6} \oplus 4 \pi_{7} \oplus 4 \pi_{8}\right] .
$$

If $D_{3}$ is the reduced orbit of a point with stabilizer $H_{3}$, then $D_{3}$ is non-special and

$$
\begin{gathered}
{\left[\operatorname{deg}_{e q}\left(D_{3}\right)\right]=\left[\pi_{\theta_{3}^{10}}\right]=\left[\pi_{2} \oplus \pi_{4} \oplus \pi_{5} \oplus \pi_{6} \oplus \pi_{7} \oplus \pi_{8}\right],} \\
{\left[\operatorname{deg}_{e q}\left(2 D_{3}\right)\right]=\left[\pi_{2} \oplus \pi_{3} \oplus 2 \pi_{4} \oplus 2 \pi_{5} \oplus 2 \pi_{6} \oplus 2 \pi_{7} \oplus 2 \pi_{8}\right],}
\end{gathered}
$$

and

$$
\begin{gathered}
{\left[L\left(D_{3}\right)\right]=\left[\pi_{1} \oplus \pi_{5} \oplus \pi_{7} \oplus \pi_{8}\right],} \\
{\left[L\left(2 D_{3}\right)\right]=\left[\pi_{1} \oplus \pi_{3} \oplus \pi_{4} \oplus 2 \pi_{5} \oplus \pi_{6} \oplus 2 \pi_{7} \oplus 2 \pi_{8}\right] .}
\end{gathered}
$$




\section{Application to codes}

In this section we consider applications of our previous results to we discuss connections with the theory of error-correcting codes.

Assume that $\ell$ is a good prime. Also, assume that $k$ contains all the character values of $G$ and that $k$ is finite, where $k$ denotes the field of definition of the reduction of $X \bmod \ell$. (The point is that we want to be able to work over a separable algebraic closure $\bar{k}$ of $k$ but then be able to take $\operatorname{Gal}(\bar{k} / k)$-fixed points to obtain our results.) We recall some background on AG codes following [JT].

Let $P_{1}, \ldots, P_{n} \in X(k)$ be distinct points and $E=P_{1}+\ldots+P_{n} \in \operatorname{Div}(X)$ be stabilized by $G$. This implies that $G$ acts on the set $\operatorname{supp}(E)$ by permutation. Assume $D$ is a $G$-equivariant divisor of $X(k)$, so $G$ acts on the RiemannRoch space $L(D)$. Assume these divisors have disjoint support, $\operatorname{supp}(D) \cap$ $\operatorname{supp}(E)=\emptyset$. Let $C=C(D, E)$ denote the AG code

$$
C=\left\{\left(f\left(P_{1}\right), \ldots, f\left(P_{n}\right)\right) \mid f \in L(D)\right\} .
$$

This is the image of $L(D)$ under the evaluation map

$$
\begin{gathered}
\operatorname{eval}_{E}: L(D) \rightarrow k^{n}, \\
f \longmapsto\left(f\left(P_{1}\right), \ldots, f\left(P_{n}\right)\right) .
\end{gathered}
$$

The group $G$ acts on $C$ by $g \in G$ sending $c=\left(f\left(P_{1}\right), \ldots, f\left(P_{n}\right)\right) \in C$ to $c^{\prime}=\left(f\left(g^{-1}\left(P_{1}\right)\right), \ldots, f\left(g^{-1}\left(P_{n}\right)\right)\right)$, where $f \in L(D)$. First, we observe that this map sending $c \longmapsto c^{\prime}$, denoted $\phi(g)$, is well-defined. In other words, if $\mathrm{eval}_{E}$ is not injective and $c$ is also represented by $f^{\prime} \in L(D)$, so $c=$ $\left(f^{\prime}\left(P_{1}\right), \ldots, f^{\prime}\left(P_{n}\right)\right) \in C$, then we can easily verify $\left(f\left(g^{-1}\left(P_{1}\right)\right), \ldots, f\left(g^{-1}\left(P_{n}\right)\right)\right)=$ $\left(f^{\prime}\left(g^{-1}\left(P_{1}\right)\right), \ldots, f^{\prime}\left(g^{-1}\left(P_{n}\right)\right)\right)$. (Indeed, $G$ acts on the set $\operatorname{supp}(E)$ by permutation.) This map $\phi(g)$ induces a homomorphism of $G$ into the permutation automorphism group of the code $\operatorname{Aut}(C)$, denoted

$$
\phi: G \rightarrow \operatorname{Aut}(C)
$$

For properties of this map, see $[\mathrm{JT}]$. In particular, the following is known.

Lemma 11 If $D$ and $E$ satisfy $\operatorname{deg}(D)>2 g$ and $\operatorname{deg}(E)>2 g+2$ then $\phi$ and eval $_{E}$ are injective.

proof: We say that the space $L(D)$ separates points if for all points $P, Q \in X, f(P)=f(Q)$ (for all $f \in L(D)$ ) implies $P=Q$ (see [H] , chapter 
II, $\S 7)$. By Proposition IV.3.1 in Hartshorne [H],$D$ very ample implies $L(D)$ separates points. In general, if $L(D)$ separates points then

$$
\operatorname{Ker}(\phi)=\left\{g \in G \mid g\left(P_{i}\right)=P_{i}, 1 \leq i \leq n\right\} .
$$

It is known (proof of Prop. VII3.3, [Sti]) that if $n=\operatorname{deg}(E)>2 g+2$ then $\left\{g \in G \mid g\left(P_{i}\right)=P_{i}, 1 \leq i \leq n\right\}$ is trivial. Therefore, if $n>2 g+2$ and $L(D)$ separates points then $\phi$ is injective. Since (see Corollary IV.3.2 in Hartshorne [H] $\operatorname{deg}(D)>2 g$ implies $D$ is very ample, the lemma follows.

As an amusing application of our theory, we show how to easily recover some results of Tsafsman and Vladut on AG codes associated to modular curves.

First, we recall some notation and results from $[\mathrm{TV}]$. Let $A_{N}=\mathbb{Z}\left[\zeta_{N}, 1 / N\right]$, where $\zeta_{N}=e^{2 \pi i / N}$, let $K_{N}$ denote the quadratic subfield of $\mathbb{Q}\left(\zeta_{N}\right)$, and let $B_{N}=A_{N} \cap K_{N}$. There is a scheme $X(N) / \mathbb{Z}[1 / N]$ which represents a moduli functor "parameterizing" elliptic curves $E$ with a level $N$ structure $\alpha_{N}$. There is a scheme $X_{P}(N) / \mathbb{Z}[1 / N]$ which represents a moduli functor "parameterizing" elliptic curves $E$ with a "projective" level $N$ structure $\beta_{N}$. If $P$ is a prime ideal in the ring of integers $\mathcal{O}_{K_{N}}$ dividing $\ell$ then the reduction of the form of $X(N)$ defined over $K_{N}$, denoted $X(N) / P$, is a smooth projective absolutely irreducible curve over the residue field $k(P)$, with a $P S L_{2}(\mathbb{Z} / N \mathbb{Z})$-action commuting with the reduction. Similarly, with $X(N)$ replaced by $X_{P}(N)$. Recall from $\S 4.1 .3$ of [TV] that

$$
k(P)= \begin{cases}G F\left(\ell^{2}\right), & (\ell)=P, \\ G F(\ell), & (\ell)=P P^{\prime},\end{cases}
$$

Let $X_{N}^{\prime}=X_{P}(N) / P$ and let

$$
\psi_{N}^{\prime}: X_{N}^{\prime} \rightarrow X_{N}^{\prime} / P S L_{2}(\mathbb{Z} / N \mathbb{Z}) \cong \mathbb{P}^{1}
$$

denote the quotient map. Let $D_{\infty}$ denote the reduced orbit (in the sense of Borne) of the point $\infty$, so $\operatorname{deg}\left(D_{\infty}\right)=|G| / N$. Let $D=r D_{\infty}$, for $r \geq 1$. According to [TV], in general, this divisor is actually defined over $G F(\ell)$, not just $k(P)$. Moreover, $\operatorname{deg}(D)=r \cdot\left(N^{2}-1\right) / 2$. Let $E=P_{1}+\ldots+P_{n}$ be the sum of all the supersingular points of $X_{N}^{\prime}$ and let

$$
C=C\left(X_{N}^{\prime}, E, D\right)=\left\{\left(f\left(P_{1}\right), \ldots, f\left(P_{n}\right)\right) \mid f \in L(D)\right\}
$$


denote the AG code associated to $X_{N}^{\prime}, D, E$. This is a $G$-module, via (19). Moreover, choosing $r$ suitably yields a "good" family of codes with large automorphism group.

In fact, if $D$ is "sufficiently large" (so, $D$ is non-special and both $\phi$, eval $_{E}$ are injective) then the Brauer-character analoguess of formulas in section 6 give not only the $G$-module structure of each $L\left(r D_{\infty}\right)$, but that of $C$ as well.

See also Remark 4.1.66 in [TV].

\section{$9 \quad$ AG codes associated to $X(7)$}

We focus on the Klein quartic example started in [JK]. We also use Elkies E] as a general reference.

Let $k=G F(43)$. This field contains $7^{\text {th }}$ roots of unity $\left(\zeta_{7}=41\right)$, cube roots of unity $\left(\zeta_{3}=36\right)$, and the square root of $-7($ take $\sqrt{-7}=6)$. Consider

$$
\begin{gathered}
\rho_{1}=\left(\begin{array}{ccc}
\zeta_{7}^{4} & 0 & 0 \\
0 & \zeta_{7}^{2} & 0 \\
0 & 0 & \zeta_{7}
\end{array}\right)=\left(\begin{array}{ccc}
16 & 0 & 0 \\
0 & 4 & 0 \\
0 & 0 & 41
\end{array}\right), \\
\rho_{2}=\left(\begin{array}{lll}
0 & 1 & 0 \\
0 & 0 & 1 \\
1 & 0 & 0
\end{array}\right),
\end{gathered}
$$

and

$$
\begin{aligned}
\rho_{3}= & \left(\begin{array}{ccc}
\left(\zeta_{7}-\zeta_{7}{ }^{6}\right) / \sqrt{-7} & \left(\zeta_{7}^{2}-\zeta_{7}^{5}\right) / \sqrt{-7} & \left(\zeta_{7}^{4}-\zeta_{7}^{3}\right) / \sqrt{-7} \\
\left(\zeta_{7}^{2}-\zeta_{7}^{5}\right) / \sqrt{-7} & \left(\zeta_{7}^{4}-\zeta_{7}^{3}\right) / \sqrt{-7} & \left(\zeta_{7}-\zeta_{7}^{6}\right) / \sqrt{-7} \\
\left(\zeta_{7}^{4}-\zeta_{7}^{3}\right) / \sqrt{-7} & \left(\zeta_{7}-\zeta_{7}^{6}\right) / \sqrt{-7} & \left(\zeta_{7}^{2}-\zeta_{7}{ }^{5}\right) / \sqrt{-7}
\end{array}\right) \\
= & \left(\begin{array}{ccc}
11 & 37 & 39 \\
37 & 39 & 11 \\
39 & 11 & 37
\end{array}\right) .
\end{aligned}
$$

It may be checked that these matrices preserve the form

$$
\phi(x, y, z)=x^{3} y+y^{3} z+z^{3} x,
$$

over $k$. They generate the subgroup $G \cong P S L_{2}(7)$ of order 168 in $P G L(3, k)$. The Klein curve $x^{3} y+y^{3} z+z^{3} x=0$, denoted here by $X$, has no other automorphisms in characteristic 43 , so $G=A u t_{k}(X)$. 
Let $D_{\infty}$ denote the reduced orbit of the point $\infty$, so $\operatorname{deg}\left(D_{\infty}\right)=|G| / N=$ 24 , and let $D=r D_{\infty}$. Let $E=P_{1}+\ldots+P_{n}$ denote the sum of the remaining $k(P)$-rational points of $X$, so $D$ and $E$ have disjoint support.

If $C$ is as in (18) then then map $\phi$ in (19) is injective. Since $e v a l_{E}$ is injective as well, the $G$-module structure of $C$ is the same as that of $L(D)$, which is known thanks to the Brauer-character analog of the formula (14). See $\$ 7.1$ above and also Example 3, JK.

Magma tells us that the points of $X(k)$ are

$$
\begin{gathered}
\{(0: 1: 0),(0: 0: 1),(1: 0: 0),(19: 9: 1),(36: 9: 1),(31: 9: 1), \\
(19: 27: 1),(1: 38: 1),(27: 38: 1),(15: 38: 1),(12: 28: 1),(38: 28: 1), \\
(36: 28: 1),(40: 41: 1),(10: 25: 1),(20: 25: 1),(13: 25: 1), \\
(20: 32: 1),(42: 10: 1),(35: 10: 1),(9: 10: 1),(40: 30: 1), \\
(13: 30: 1),(33: 30: 1),(24: 4: 1),(25: 36: 1),(12: 36: 1),(6: 36: 1), \\
(12: 22: 1),(14: 23: 1),(8: 23: 1),(21: 23: 1),(24: 26: 1),(37: 26: 1), \\
(25: 26: 1),(23: 35: 1),(15: 14: 1),(33: 14: 1),(38: 14: 1),(33: 42: 1), \\
(17: 40: 1),(4: 40: 1),(22: 40: 1),(5: 34: 1),(15: 34: 1),(23: 34: 1), \\
(31: 16: 1),(40: 15: 1),(37: 15: 1),(9: 15: 1),(37: 2: 1),(11: 6: 1), \\
(39: 6: 1),(36: 6: 1),(31: 18: 1),(9: 18: 1),(3: 18: 1),(10: 11: 1), \\
(38: 14: 1),(33: 42: 1), \\
(17: 40: 1),(4: 40: 1),(22: 40: 1),(5: 34: 1),(15: 34: 1),(23: 34: 1), \\
(31: 16: 1),(40: 15: 1),(37: 15: 1),(9: 15: 1),(37: 2: 1),(11: 6: 1), \\
(39: 6: 1),(36: 6: 1),(31: 18: 1),(9: 18: 1),(3: 18: 1),(10: 11: 1), \\
(5: 13: 1),(24: 13: 1),(14: 13: 1),(5: 39: 1),(41: 31: 1), \\
(13: 31: 1),(32: 31: 1),(10: 7: 1),(14: 7: 1),(19: 7: 1),(6: 21: 1), \\
(17: 17: 1),(3: 17: 1),(23: 17: 1),(3: 8: 1),(25: 24: 1), \\
(16: 24: 1),(2: 24: 1),(20: 29: 1),(17: 29: 1),(6: 29: 1),(38: 1: 1)\}
\end{gathered}
$$

Magma tells us that the orbit of $(1: 0: 0) \in X(k)$ under $G$ is

$$
\begin{gathered}
\{(1: 0: 0),(36: 28: 1),(32: 31: 1),(15: 34: 1),(9: 18: 1),(37: 2: 1), \\
(2: 24: 1),(3: 8: 1),(22: 40: 1),(19: 27: 1),(13: 30: 1), \\
(5: 39: 1),(8: 23: 1),(14: 7: 1),(25: 26: 1),(33: 42: 1), \\
(17: 29: 1),(42: 10: 1),(0: 10),(0: 0: 1), \\
(20: 32: 1),(12: 22: 1),(27: 38: 1),(39: 6: 1)\}
\end{gathered}
$$

We evaluate each element of the Riemann-Roch space $L(D)$ at a point in the complement of the above-mentioned orbit in the set of rational points $X(k)$. For concreteness, a typical basis element of $L(D)$, when $r=1$, looks like 


$$
\begin{gathered}
y^{j} x^{i}(y+1)^{-1}(y+3)^{-1}(y+4)^{-1}(y+5)^{-1}(y+9)^{-1}(y+11)^{-1}(y+12)^{-1}(y+13)^{-1} \times \\
\times(y+14)^{-1}(y+15)^{-1}(y+16)^{-1}(y+17)^{-1}(y+19)^{-1}(y+20)^{-1}(y+21)^{-1} \times \\
\times(y+25)^{-1}(y+33)^{-1}(y+35)^{-1}(y+36)^{-1}(y+37)^{-1}(y+41)^{-1} \times \\
\times\left(x^{2} y^{8}+34 x^{2} y+16 x y^{11}+2 x y^{4}+17 y^{14}+33 y^{7}+17\right)
\end{gathered}
$$

for example with $i=2$ and $j=6$. In fact, all such elements with $i=1,2$ and $0 \leq j \leq 6$ are basis elements of $L(D)$.

With $r=1$, Magma says that $C=C(X, D, E)$ is a $[56,22,32] \operatorname{code}^{2}$. It has a generator matrix of in standard form $(I \mid A)$, where $I$ is the $22 \times 22$ identity and $A$ can be given explicitly as well. In this case, $e v a l_{E}$ is injective.

With $r=2$, Magma says that $C=C(X, D, E)$ is a $[56,46,8]$ code. It has a generator matrix of in standard form $(I \mid A)$, where $I$ is the $46 \times 46$ identity and $A$ can be given explicitly as well. In this case, eval $_{E}$ is injective.

With $r=3$, Magma says that $C=C(X, D, E)$ is a $[56,56,1]$ code. In this case, eval $E$ is not injective. Indeed, $\operatorname{dim} L\left(3 D_{\infty}\right)=70$.

Remark 2 Indeed, it is known more generally, that for an $A G$ code constructed as above from a curve of genus $g$ that $n \leq \operatorname{dim}(C)+d(C)+g-1$, where $d(C)$ denotes the minimum distance (Theorem 3.1.1 in [TV]). Therefore, as an $A G$ code, the codes constructed above with $r=1,2$ are as "long as possible". Are they best possible over GF(43) for those $k, d$ ?

Remark 3 An attempt to compute the permutation automorphism group of the above [56,22,32] code using MAGMA failed due to lack of memory (on an AMD64 linux machine with $1.5 \mathrm{G}$ of $R A M)$.

Remark 4 In general, $\S 4.1$ of $[T V]$ shows how to construct a family of "good" codes from the curves $X=X_{N}^{\prime}$, for $N>5$ is a prime, with automorphism group $G=P S L(2, p)$.

\section{Appendix: Tables for Theorem 8}

The following tables are an intermediate step in the proof of theorem 8 . They were obtained by compiling the results in $\S \$ 4.14 .3$.

\footnotetext{
${ }^{2}$ In other words, $C$ has length 56 , dimension 22 over $k$, and minimum distance 32 .
} 


\begin{tabular}{|c|c|c|c|}
\hline$N \equiv 1 \quad(\bmod 24)$ & $\operatorname{Ind}_{H_{1}}^{G} \theta_{1}$ & $\operatorname{Ind}_{H_{2}}^{G}\left(\theta_{2}+2 \theta_{2}^{2}\right)$ & $\operatorname{Ind}_{H_{3}}^{G}\left(\sum_{\ell=1}^{N-1} \ell \theta_{3}^{\ell}\right)$ \\
\hline $\mathbf{1}_{G}$ & 0 & 0 & 0 \\
$W^{\prime}$ & $\frac{N-1}{4}$ & $\frac{N-1}{2}$ & $S_{\mathcal{Q}}$ \\
$W^{\prime \prime}$ & $\frac{N-1}{4}$ & $N-1$ & $S_{\mathcal{N}}$ \\
$W_{\alpha}$ such that & $\frac{N-1}{2}$ & $\frac{N(N-1)}{2}$ \\
$\alpha(i)=\alpha(\omega)=1$ & $\frac{N-1}{2}$ & $N+2$ & $\frac{N(N-1)}{2}$ \\
$W_{\alpha}$ such that & & $\frac{N(N-1)}{2}$ \\
$\alpha(i)=1, \alpha(\omega) \neq 1$ & $\frac{N+3}{2}$ & $N-1$ & $\frac{N(N-1)}{2}$ \\
$W_{\alpha}$ such that & & $\frac{N(N-1)}{2}$ \\
$\alpha(i)=-1, \alpha(\omega)=1$ & $\frac{N+3}{2}$ & $N+2$ & $\frac{N(N-1)}{2}$ \\
$W_{\alpha}$ such that & $\frac{N-1}{2}$ & $N-1$ & $N-1$ \\
$\alpha(i)=-1, \alpha(\omega) \neq 1$ & $\frac{N-1}{2}$ & $N-1$ & \\
$X_{\beta}$ & & & \\
$V$ & & & \\
\hline
\end{tabular}

\begin{tabular}{|c|c|c|c|}
\hline$N \equiv 5 \quad(\bmod 24)$ & $\operatorname{Ind}_{H_{1}}^{G} \theta_{1}$ & $\operatorname{Ind}_{H_{2}}^{G}\left(\theta_{2}+2 \theta_{2}^{2}\right)$ & $\operatorname{Ind}_{H_{3}}^{G}\left(\sum_{\ell=1}^{N-1} \ell \theta_{3}^{\ell}\right)$ \\
\hline $\mathbf{1}_{G}$ & 0 & 0 & 0 \\
$W^{\prime}$ & $\frac{N+3}{4}$ & $\frac{N+1}{2}$ & $S_{\mathcal{Q}}$ \\
$W^{\prime \prime}$ & $\frac{N+3}{2}$ & $\frac{N^{\prime}}{2}$ & $S_{\mathcal{N}}$ \\
$W_{\alpha}$ such that & $\frac{N-1}{2}$ & $N+1$ & $\frac{N(N-1)}{2}$ \\
$\alpha(i)=1$ & $\frac{N+3}{2}$ & $N+1$ & $\frac{N(N-1)}{2}$ \\
$W_{\alpha}$ such that \\
$\alpha(i)=-1$ \\
$X_{\beta}$ such that \\
$\beta(\omega)=1$ & $\frac{N-1}{2}$ & $N+1$ & $\frac{N(N-1)}{2}$ \\
$X_{\beta}$ such that & $\frac{N-1}{2}$ & $N-2$ & $\frac{N(N-1)}{2}$ \\
$\beta(\omega) \neq 1$ & $\frac{N-1}{2}$ & $N+1$ & $\frac{N(N-1)}{2}$ \\
$V$ &
\end{tabular}




\begin{tabular}{|c|c|c|c|}
\hline$N \equiv 7 \quad(\bmod 24)$ & $\operatorname{Ind}_{H_{1}}^{G} \theta_{1}$ & $\operatorname{Ind}_{H_{2}}^{G}\left(\theta_{2}+2 \theta_{2}^{2}\right)$ & $\operatorname{Ind}_{H_{3}}^{G}\left(\sum_{\ell=1}^{N-1} \ell \theta_{3}^{\ell}\right)$ \\
\hline $\begin{array}{c}\mathbf{1}_{G} \\
W_{\alpha} \text { such that } \\
\alpha(\omega)=1\end{array}$ & 0 & 0 & 0 \\
$W_{\alpha}$ such that & $\frac{N+1}{2}$ & $N-1$ & $\frac{N(N-1)}{2}$ \\
$\alpha(\omega) \neq 1$ & $\frac{N+1}{2}$ & $N+2$ & $\frac{N(N-1)}{2}$ \\
$X^{\prime}$ & $\frac{N+1}{4}$ & $\frac{N-1}{2}$ & $S_{\mathcal{Q}}$ \\
$X^{\prime \prime}$ & $\frac{N+1}{4}$ & $\frac{N-1}{2}$ & $S_{\mathcal{N}}$ \\
$X_{\beta}$ such that & $\frac{N+1}{2}$ & $N-1$ & $\frac{N(N-1)}{2}$ \\
$\beta(i)=1$ & $\frac{N-3}{2}$ & $N-1$ & $\frac{N(N-1)}{2}$ \\
$X_{\beta}$ such that & $N-1$ & $\frac{N(N-1)}{2}$ \\
$\beta(i)=-1$ & $\frac{N+1}{2}$ & $N-1$ \\
$V$
\end{tabular}

\begin{tabular}{|c|c|c|c|}
\hline$N \equiv 11 \quad(\bmod 24)$ & $\operatorname{Ind}_{H_{1}}^{G} \theta_{1}$ & $\operatorname{Ind}_{H_{2}}^{G}\left(\theta_{2}+2 \theta_{2}^{2}\right)$ & $\operatorname{Ind}_{H_{3}}^{G}\left(\sum_{\ell=1}^{N-1} \ell \theta_{3}^{\ell}\right)$ \\
\hline $\mathbf{1}_{G}$ & 0 & 0 & 0 \\
$W_{\alpha}$ & $\frac{N+1}{2}$ & $N+1$ & $\frac{N(N-1)}{2}$ \\
$X^{\prime}$ & $\frac{N_{-3}}{S_{\mathcal{Q}}}$ & $\frac{N+1}{2}$ & $S_{\mathcal{N}}$ \\
$X^{\prime \prime}$ & $\frac{N-3}{2}$ & $\frac{N(N-1)}{2}$ \\
$X_{\beta}$ such that & $\frac{N+1}{2}$ & $N+1$ & $\frac{N(N-1)}{2}$ \\
$\beta(i)=\beta(\omega)=1$ & $\frac{N+1}{2}$ & $N-2$ & $\frac{N(N-1)}{2}$ \\
$X_{\beta}$ such that & & $\frac{N(N-1)}{2}$ \\
$\beta(i)=1, \beta(\omega) \neq 1$ & $\frac{N-3}{2}$ & $N+1$ & $\frac{N(N-1)}{2}$ \\
$X_{\beta}$ such that & $\frac{N-3}{2}$ & $N-2$ & \\
$\beta(i)=-1, \beta(\omega)=1$ & $\frac{N+1}{2}$ & $N+1$ & \\
$X_{\beta}$ such that & & & \\
$\beta(i)=-1, \beta(\omega) \neq 1$ & & & \\
$V$ & & & \\
\hline
\end{tabular}




\begin{tabular}{|c|c|c|c|}
\hline$N \equiv 13 \quad(\bmod 24)$ & $\operatorname{Ind}_{H_{1}}^{G} \theta_{1}$ & $\operatorname{Ind}_{H_{2}}^{G}\left(\theta_{2}+2 \theta_{2}^{2}\right)$ & $\operatorname{Ind}_{H_{3}}^{G}\left(\sum_{\ell=1}^{N-1} \ell \theta_{3}^{\ell}\right)$ \\
\hline $\mathbf{1}_{G}$ & 0 & 0 & 0 \\
$W^{\prime}$ & $\frac{N+3}{4}$ & $\frac{N-1}{2}$ & $S_{\mathcal{Q}}$ \\
$W^{\prime \prime}$ & $\frac{N+3}{4}$ & $\frac{N^{2}}{2}$ & $S_{\mathcal{N}}$ \\
$W_{\alpha}$ such that & $\frac{N-1}{2}$ & $N-1$ & $\frac{N(N-1)}{2}$ \\
$\alpha(i)=\alpha(\omega)=1$ & $\frac{N-1}{2}$ & $N+2$ & $\frac{N(N-1)}{2}$ \\
$W_{\alpha}$ such that \\
$\alpha(i)=1, \alpha(\omega) \neq 1$ & $\frac{N+3}{2}$ & $N-1$ & $\frac{N(N-1)}{2}$ \\
$W_{\alpha}$ such that & & $\frac{N(N-1)}{2}$ \\
$\alpha(i)=-1, \alpha(\omega)=1$ & $\frac{N+3}{2}$ & $N+2$ & $\frac{N(N-1)}{2}$ \\
$W_{\alpha}$ such that & $\frac{N-1}{2}$ & $N-1$ & $\frac{N(N-1)}{2}$ \\
$\alpha(i)=-1, \alpha(\omega) \neq 1$ & $\frac{N-1}{2}$ & $N-1$ & \\
$X_{\beta}$ & & & \\
$V$ & & & \\
\hline
\end{tabular}

\begin{tabular}{|c|c|c|c|}
\hline$N \equiv 17 \quad(\bmod 24)$ & $\operatorname{Ind}_{H_{1}}^{G} \theta_{1}$ & $\operatorname{Ind}_{H_{2}}^{G}\left(\theta_{2}+2 \theta_{2}^{2}\right)$ & $\operatorname{Ind}_{H_{3}}^{G}\left(\sum_{\ell=1}^{N-1} \ell \theta_{3}^{\ell}\right)$ \\
\hline $\mathbf{1}_{G}$ & 0 & 0 & 0 \\
$W^{\prime}$ & $\frac{N-1}{4}$ & $\frac{N+1}{2}$ & $S_{\mathcal{Q}}$ \\
$W^{\prime \prime}$ & $\frac{N-1}{2}$ & $\frac{N+1}{2}$ & $S_{\mathcal{N}}$ \\
$W_{\alpha}$ such that & $\frac{N-1}{2}$ & $N+1$ & $\frac{N(N-1)}{2}$ \\
$\alpha(i)=1$ & $\frac{N+3}{2}$ & $N+1$ & $\frac{N(N-1)}{2}$ \\
$W_{\alpha}$ such that \\
$\alpha(i)=-1$ & $\frac{N-1}{2}$ & $N+1$ & $\frac{N(N-1)}{2}$ \\
$X_{\beta}$ such that & & $\frac{N(N-1)}{2}$ \\
$\beta(\omega)=1$ & $\frac{N-1}{2}$ & $N-2$ & $\frac{N(N-1)}{2}$ \\
$X_{\beta}$ such that & $N+1$ & \\
$\beta(\omega) \neq 1$ & $\frac{N-1}{2}$ & $N+1$ & \\
$V$ & & & \\
\hline
\end{tabular}




\begin{tabular}{|c|c|c|c|}
\hline$N \equiv 19 \quad(\bmod 24)$ & $\operatorname{Ind}_{H_{1}}^{G} \theta_{1}$ & $\operatorname{Ind}_{H_{2}}^{G}\left(\theta_{2}+2 \theta_{2}^{2}\right)$ & $\operatorname{Ind}_{H_{3}}^{G}\left(\sum_{\ell=1}^{N-1} \ell \theta_{3}^{\ell}\right)$ \\
\hline $\begin{array}{c}\mathbf{1}_{G} \\
W_{\alpha} \text { such that } \\
\alpha(\omega)=1 \\
W_{\alpha} \text { such that } \\
\alpha(\omega) \neq 1\end{array}$ & $\frac{N+1}{2}$ & $N-1$ & 0 \\
$\frac{N+1}{2}$ & $N+2$ & $\frac{N(N-1)}{2}$ \\
$X^{\prime}$ & $\frac{N-3}{4}$ & $\frac{N-1}{2}$ & $\frac{N(N-1)}{2}$ \\
$X^{\prime \prime}$ & $\frac{N-3}{4}$ & $\frac{N-1}{2}$ & $S_{\mathcal{N}}$ \\
$X_{\beta}$ such that & $\frac{N+1}{2}$ & $N-1$ & $\frac{N(N-1)}{2}$ \\
$X_{\beta}$ such that & $\frac{N-3}{2}$ & $N-1$ & $\frac{N(N-1)}{2}$ \\
$\beta(i)=-1$ & $\frac{N+1}{2}$ & $N-1$ & $\frac{N(N-1)}{2}$ \\
$V$ & & & \\
\hline
\end{tabular}

\begin{tabular}{|c|c|c|c|}
\hline$N \equiv 23 \quad(\bmod 24)$ & $\operatorname{Ind}_{H_{1}}^{G} \theta_{1}$ & $\operatorname{Ind}_{H_{2}}^{G}\left(\theta_{2}+2 \theta_{2}^{2}\right)$ & $\operatorname{Ind}_{H_{3}}^{G}\left(\sum_{\ell=1}^{N-1} \ell \theta_{3}^{\ell}\right)$ \\
\hline $\mathbf{1}_{G}$ & 0 & 0 & 0 \\
$W_{\alpha}$ & $\frac{N+1}{2}$ & $N+1$ & $\frac{N(N-1)}{2}$ \\
$X^{\prime}$ & $\frac{N+1}{4}$ & $\frac{N+1}{2}$ & $S_{\mathcal{Q}}$ \\
$X^{\prime \prime}$ & $\frac{N+1}{4}$ & $\frac{N+1}{2}$ & $\frac{N(N-1)}{2}$ \\
$X_{\beta}$ such that & $\frac{N+1}{2}$ & $N+1$ & $\frac{N(N-1)}{2}$ \\
$\beta(i)=\beta(\omega)=1$ & $\frac{N+1}{2}$ & $N-2$ & $\frac{N(N-1)}{2}$ \\
$X_{\beta}$ such that & & $\frac{N(N-1)}{2}$ \\
$\beta(i)=1, \beta(\omega) \neq 1$ & $\frac{N-3}{2}$ & $N+1$ & $\frac{N(N-1)}{2}$ \\
$X_{\beta}$ such that & $N-2$ & \\
$\beta(i)=-1, \beta(\omega)=1$ & $\frac{N-3}{2}$ & $N+1$ & $\frac{N+1}{2}$ \\
$X_{\beta}$ such that & $N(i)=-1, \beta(\omega) \neq 1$ & & \\
$V$ & & & \\
\hline
\end{tabular}




\section{Appendix: Ramification modules and GAP code}

We used GAP to compute the ramification module for many small values of $N$. Here are the first results:

\begin{tabular}{|c|c|c|}
\hline$N$ & $\begin{array}{c}G \text {-module structure of } \tilde{\Gamma_{G}} \\
\text { by definition }\end{array}$ & $\begin{array}{c}G \text {-module structure of } \tilde{\Gamma_{G}} \\
\text { using JK formula }\end{array}$ \\
\hline 5 & {$[0,3,3,4,5]$} & {$[0,3,3,4,5]$} \\
7 & {$[0,3,4,6,7,8]$} & {$[0,7 / 2,7 / 2,6,7,8]$} \\
11 & {$[0,5,6,11,10,12,12,12]$} & {$[0,11 / 2,11 / 2,11,10,12,12,12]$} \\
13 & {$[0,7,7,13,13,13,13,14,15]$} & {$[0,7,7,13,13,13,13,14,15]$} \\
17 & {$[0,9,9,18,17,17,17,18, \ldots]$} & {$[0,9,9,18,17,17,17,18, \ldots]$} \\
19 & {$[0,9,10,20,20,19,19,20, \ldots]$} & {$[0,19 / 2,19 / 2,20,20,19,19,20, \ldots]$} \\
23 & {$[0,11,14,24,24,24,23,23, \ldots]$} & {$[0,25 / 2,25 / 2,24,24,24,23,23, \ldots]$} \\
29 & {$[0,16,16,30,31,31,30, \ldots]$} & {$[0,16,16,30,31,31,30, \ldots]$} \\
\hline
\end{tabular}

GAP was able to go up to and including $N=277$ (in which case $G$ is order about 20 million).

GAP code for the tables

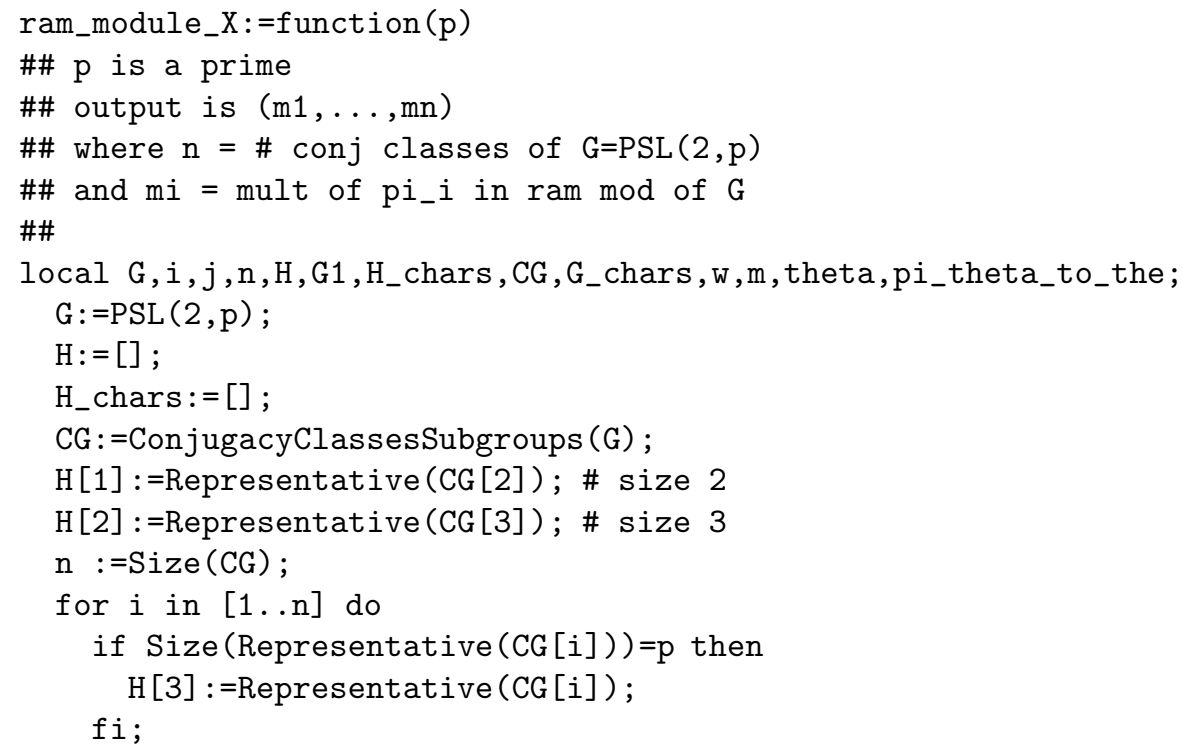




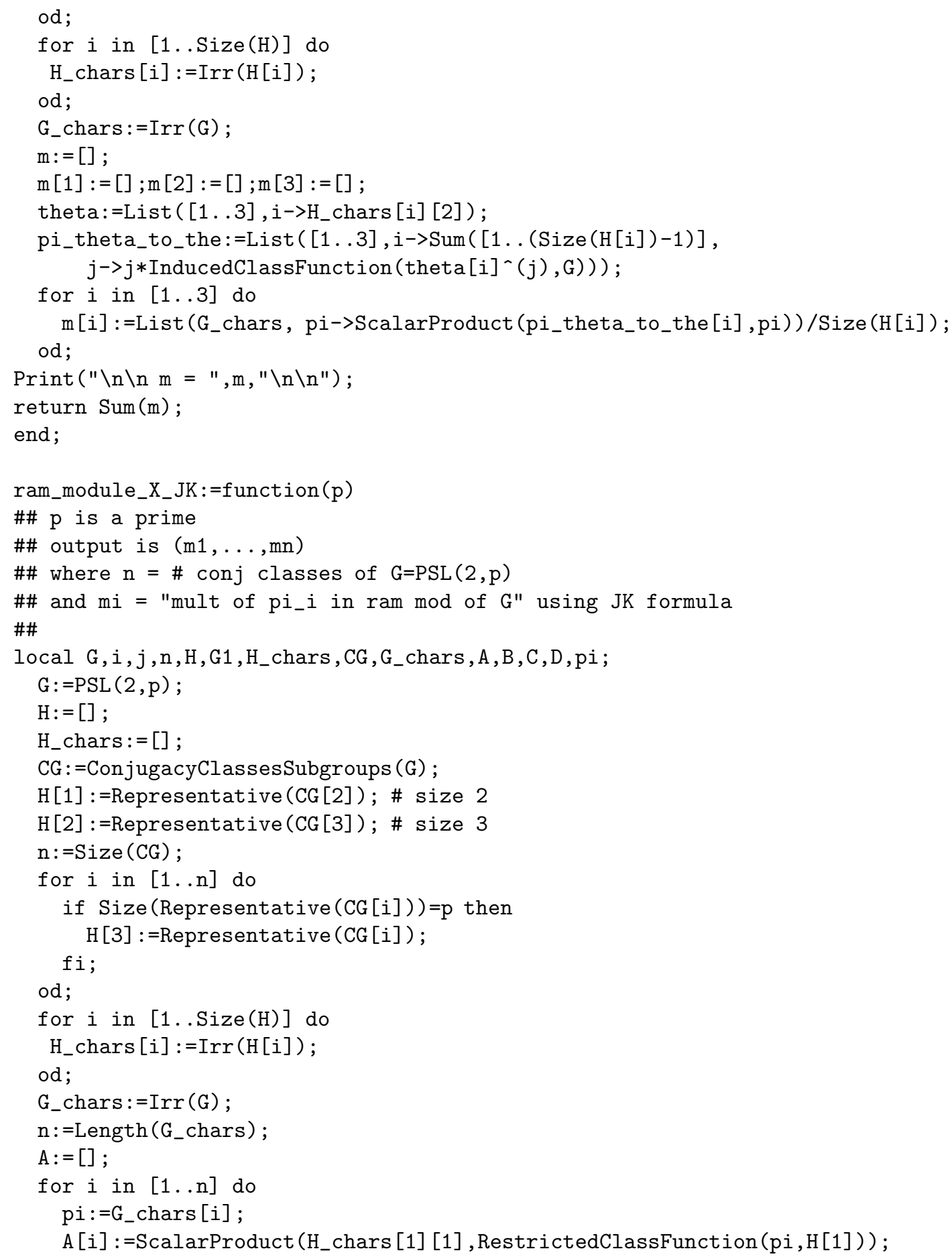




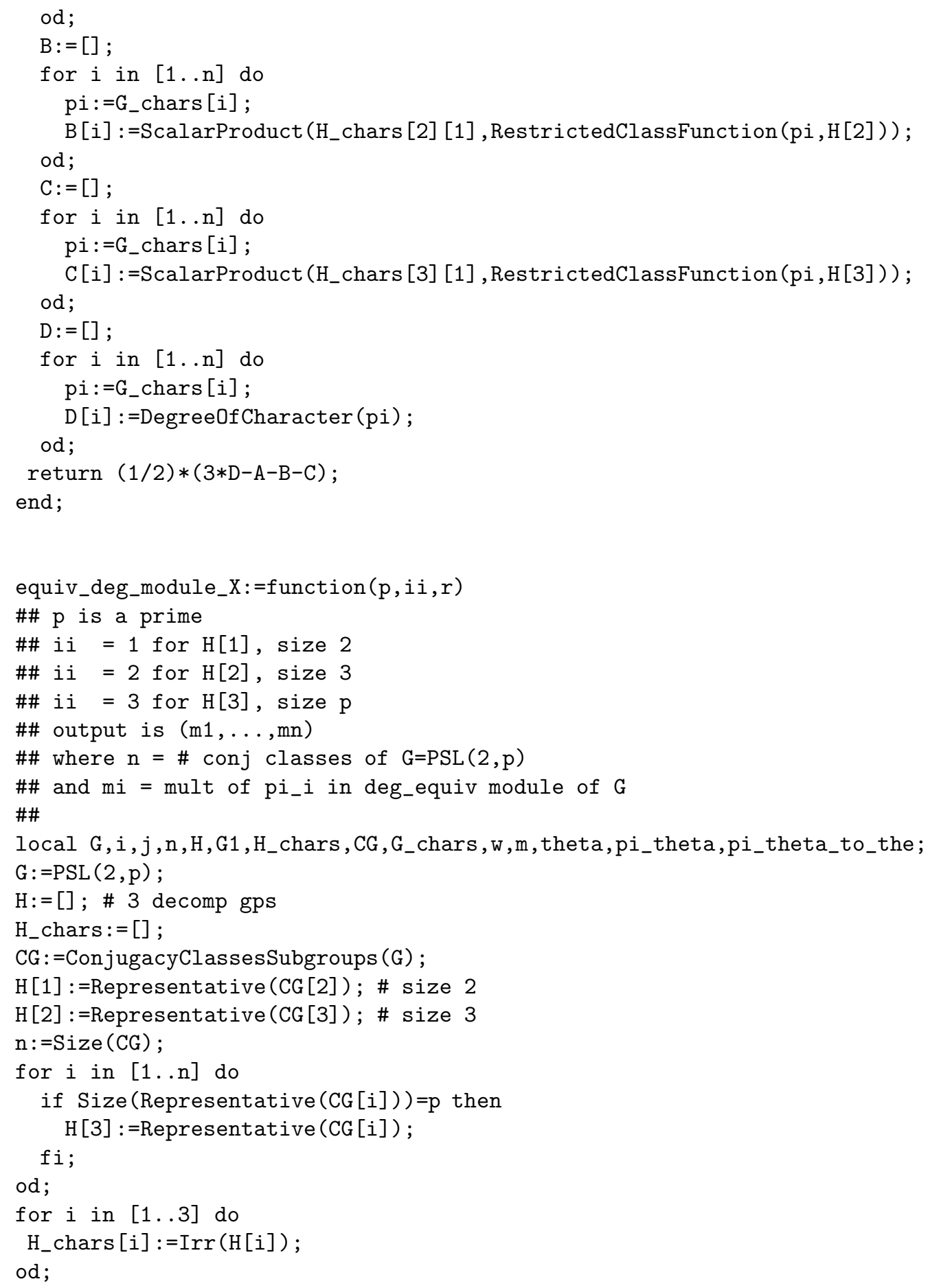




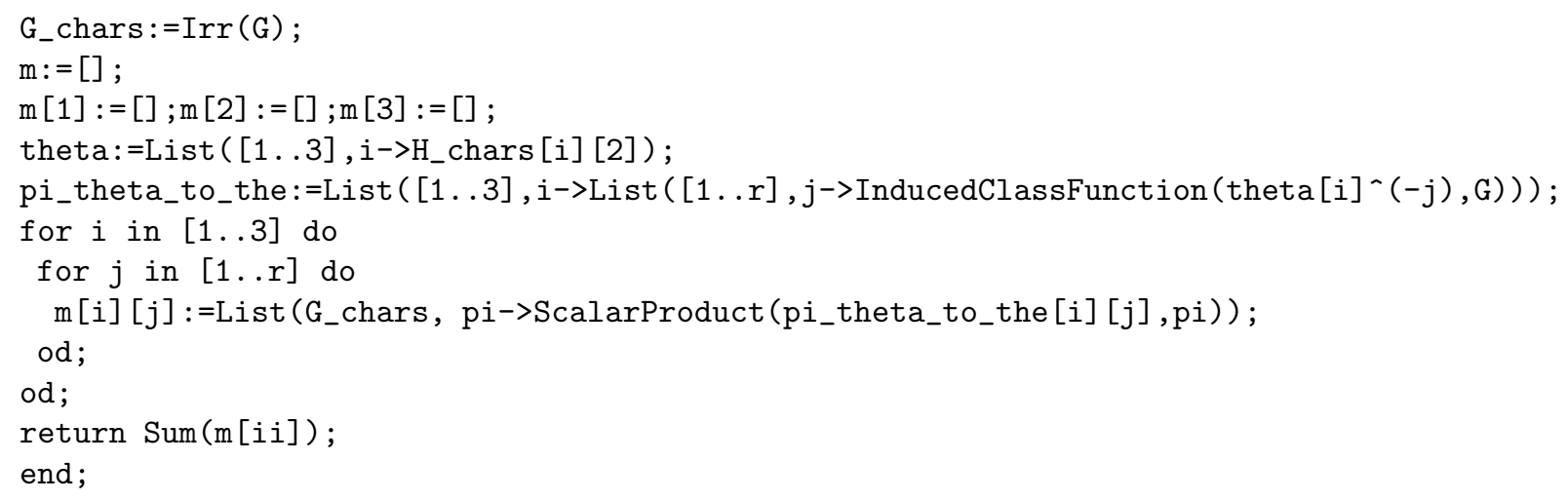

\section{References}

[A1] A. Adler, "The Mathieu group $M_{11}$ and the modular curve $X_{11}$," Proc. London Math Society, $\underline{74}(1997) 1-28$.

See also the preprint " $X(11)$ and $M_{11}$," available at http://swiss.csail.mit.edu/ adler/ARTICLES/

[A2] — , "Some integral representations of $P S L_{2}\left(\mathbb{F}_{p}\right)$ and their applications," J. Algebra 72(1981)115-145.

[BCG] P. Bending, A. Camina, R. Guralnick "Automorphisms of the modular curve $X(p)$ in positive characteristic," 2003 preprint.

[BZ] I. Berkovich, E. Zhmud, Characters of Finite Groups, vol 2. AMS, 1999.

[B] N. Borne, "Une formule de Riemann-Roch equivariante pour des courbes," thesis, Univ. Bordeaux, 1999. Available on the web at http://www.dm.unibo.it/ borne/

[E] N. Elkies, "The Klein quartic in number theory," in The eightfold way, MSRI Publ. vol 38, 1998.

[FH] W. Fulton and J. Harris, Representation theory: a first course, Springer-Verlag, 1991. 
[GAP] The GAP Group, GAP - Groups, Algorithms, and Programming, Version 4.4; 2002, (\protect \vrule width0pt \protect \href\{http://www.gap-system.org\}\{http://www.ga

[H] R. Hartshorne, Algebraic geometry, Springer-Verlag, 1977.

[Ja] G. Janusz, "Simple components of $\mathbb{Q}[S L(2, q)]$," Comm. Alg. 1(1974)122.

[JK] D. Joyner and A. Ksir, "Representations of finite groups on Riemann-Roch spaces, II," preprint 2003, available at http://front.math.ucdavis. edu/math. AG/0312383

[JT] D. Joyner and W. Traves, "Representations of finite groups on Riemann-Roch spaces," preprint, 2002 available at http://front.math.ucdavis.edu/math.AG/0210408 (revised version posted April, 2004)

[KP] C. Khare and D. Prasad, "Extending local representations to global representations," Kyoto Journal of Mathematics, $\underline{36}$ (1996)471-480

[MAGMA] W. Bosma, J. Cannon, C. Playoust, "The MAGMA algebra system, I: The user language," J. Symb. Comp., 244(1997)235-265.

(See also the MAGMA homepage at http://www.maths.usyd.edu.au:8000/u/magma/).

[MMN] H. Martin, J. Migliore, S. Nollet, "Degrees of generators of ideals defining curves in projective space," Comm. Alg.26 (1998)1209-1231.

[N] S. Nakajima, "Galois module structure of cohomology groups for tamely ramified coverings of algebraic varieties," J. Number Theory 22 (1986) 115-123.

[R1] C. Ritzenthaler, "Problèmes arithmétiques relatifs à certaines familles de courbes sur les corps finis," Thesis, Univ. Paris 7, 2003.

[R2] — - "Action du groupe de Mathieu $M_{11}$ sur la courbe modulaire $X(11)$ en caractéristique 3," Masters thesis, Univ. Paris 6, 1998.

[R3] — - "Automorphismes des courbes modulaires $X(n)$ en caractristique p," Manuscripta Math. 109(2002)49 - 62 . 
[Sc] C. Schoen, "On certain modular representations in the cohomology of algebraic curves," J. Alg. 135(1990)1-18.

[S] G. Shimura, Arithmetic theory of automorphic functions, Princeton Univ. Press, 1971.

[Sti] H. Stichtenoth, Algebraic function fields and codes, SpringerVerlag, 1993.

[TV] M. A. Tsfasman and S. G. Vladut, Algebraic-geometric codes, Mathematics and its Applications, Kluwer Academic Publishers, Dordrechet 1991.

[V] J. Velu, "Courbes elliptiques munies dun sous-groupe $\mathbb{Z} / n \mathbb{Z} \times \mu_{n}$," Bull. Soc. Math. France, Memoire, 1978. 